\title{
KINEMATIC COMPATIBILITY CONDITIONS OF THIRD ORDER FOR A THREE-DIMENSIONAL SURFACE VECTOR FIELD
}

\author{
BY
}

\author{
R. S. D. THOMAS
}

St. John's College and

Department of Applied Mathematics, University of Manitoba, Winnipeg, Manitoba, Canada

1. Introduction. This paper completes the extension to third order of compatibility conditions, in the sense popularized by T. Y. Thomas [11] (see also [12]), which can be used to study propagation of discontinuities of a vector field defined on a surface in euclidean space. H. Cohen and the author considered such conditions for a general tensor defined on a plane in [1]; here the tensor is restricted to be a three-dimensional vector, but the plane is replaced by a curved surface, extending the work of Cohen and Wang [6] and the author [8]. The third-order results of [1] were subsequently used to study acceleration waves in plates $[2,3]$, and it is hoped that the present conditions may be useful in similar studies, particularly on shells, where the wave may alter the shape of the shell, as [6] was used in $[4,5]$.

This section serves to introduce the topic of the paper and geometrical notation. Section two describes the kinematic notation and results drawn upon. Section three derives some commutation relations needed in subsequent sections. Section four quotes and derives directional derivatives to compare with the partial derivatives derived in Sec. five. The compatibility conditions are derived in Sec. six for trajectories normal to the singular curves, and in the final section these results are extended to trajectories oblique to those normals.

A smooth surface $S$ in euclidean space $E^{3}$ is customarily represented by three scalar functions

$$
x^{i}=x^{i}\left(\theta^{\alpha}\right) \quad(i=1,2,3 ; \alpha=1,2),
$$

where the $x^{i}$ are Cartesian coordinates indicating positions and the $\theta^{\alpha}$, Cartesian coordinates in $E^{2}$, label the material points. The summation convention will be used throughout with the ranges 1, 2, 3, for $i$ and 1, 2, for Greek. A moving surface $S_{t}$ then can be represented by making the $x^{i}$ functions of time, $x^{i}=x^{i}\left(\theta^{\alpha}, t\right)$. In vector notation, $\mathbf{x}=\mathbf{x}\left(\theta^{\alpha}, t\right)$. At each point of the surface and at each time $t$, a basis is supplied for the tangent space of $E^{3}$ by the surface's tangent-space basis $\mathbf{h}_{\alpha}=\mathbf{x}_{, \alpha}$, where we are using a 
comma to indicate partial differentiation, and $\mathbf{h}_{3}$, chosen to be perpendicular to $\mathbf{h}_{1}$ and $\mathbf{h}_{2}$ and to make $\left(\mathbf{h}_{1}, \mathbf{h}_{2}, \mathbf{h}_{3}\right)$ a right-hand system. In these terms, the metric of the surface has covariant components $h_{\alpha \beta} \equiv \mathbf{h}_{\alpha} \cdot \mathbf{h}_{\beta}$ and contravariant components $h^{\alpha \beta}$ such that $h^{\alpha \gamma} h_{\gamma \beta}=\delta_{\beta}^{\alpha}$. The reciprocal basis then is given by $\mathbf{h}^{\alpha} \equiv h^{\alpha \gamma} \mathbf{h}_{\gamma}$, and $\mathbf{h}^{3} \equiv \mathbf{h}_{3}$, and the Christoffel symbols and the second fundamental form of the surface by $\left\{\begin{array}{c}\alpha \\ \beta \gamma\end{array}\right\} \equiv \mathbf{h}^{\alpha} \cdot \mathbf{h}_{\beta, \gamma}$, $\Omega_{\alpha \beta} \equiv \mathbf{h}_{\alpha, 3} \cdot \mathbf{h}_{3}$, respectively. These relations imply that

$$
\mathbf{h}_{\alpha, \beta}=\left\{\begin{array}{c}
\gamma \\
\alpha \beta
\end{array}\right\} \mathbf{h}_{\gamma}+\Omega_{\alpha \beta} \mathbf{h}_{3}, \quad \mathbf{h}_{3, \beta}=-\Omega_{\beta}^{\alpha} \mathbf{h}_{\alpha} .
$$

The concern of this work is with a vector field $\phi$, a three-dimensional function of position on the surface, $\phi=\phi\left(\theta^{\alpha}, t\right)=\phi^{\beta} \mathbf{h}_{\beta}+\phi^{3} \mathbf{h}_{3}$, where the (contravariant) tangential and (scalar) perpendicular components of $\boldsymbol{\phi}$ are, like $\mathbf{h}_{\beta}, \mathbf{h}_{3}$, functions of position on the surface and time. It is necessary to use the covariant derivatives of the tangential components to express succinctly even the partial derivative of $\phi$. From the above relations,

$$
\phi_{. \beta}=\left(\phi_{\mid \beta}^{\alpha}-\phi^{3} \Omega_{\beta}^{\alpha}\right) \mathbf{h}_{\alpha}+\left(\phi_{. \beta}^{3}+\phi^{\alpha} \Omega_{\alpha \beta}\right) \mathbf{h}_{3} .
$$

One notes the way in which the perpendicular component influences the tangential components of the derivative and vice versa. It is the object of this paper to use the method of singular curves to find compatibility conditions that relate the jumps in the thirdorder partial or covariant derivatives involving time of the component functions of $\phi$ to the jumps in their directional derivatives, those of first and second order having been found in [6] and [8] and those of third order not involving time having been found in [9].

The method of singular curves deals with a function that is singular in the sense that it is smooth except on an oriented smooth curve $C_{t}$ moving on $S_{t}$ and that, for each $t$, it and all of its derivatives must have limits with respect to $\theta^{\alpha}$ and $t$ at $C_{t}$ from the side to which $C_{t}$ is moving (the positive side of $C_{t}$ ) and the side from which $C_{t}$ is moving (the negative side of $C_{t}$ ). The difference between these limits is called the jump in the function, denoted by square brackets, for example,

$$
\left[\phi_{, \beta}^{3}\right]=\left(\phi_{, \beta}^{3}\right)^{-}-\left(\phi_{. \beta}^{3}\right)^{+} \text {. }
$$

The order of the singularity of the function is the lowest order of partial derivative that has a nonzero jump at $C_{t}$, order zero meaning that the function itself has a jump. We shall need a formula for the jump of a product in terms of the factors; this formula is as follows:

$$
[A B]=A^{+}[B]+[A] B^{+}+[A][B] .
$$

For each $t$, we shall have occasion to require the unit tangent of $C_{t}, \mathbf{m}=d \mathbf{x} / d s$, where $s$ is the arc length along $C_{t}$ according to its given orientation. We need also, in the tangent plane of $S_{t}$, the unit normal vector $\mathbf{n}=\mathbf{h}_{3} \times \mathbf{m}$, which is generally not the normal vector of the Frenet equations. Frequently we shall have occasion to use the limits of directional derivatives of a singular function as the point of evaluation approaches $C_{t}$ and the direction approaches that of $\mathbf{m}$ or $\mathbf{n}$; when it matters whether the limit is from the positive or negative side of $C_{t}$, which one is meant will be made clear. When either 
is meant, then no indication will be made, for instance, $d \phi / d m$ will mean the limit of the directional derivative of $\phi$ in the direction $\mathbf{m}$ at $C_{t}$ from whichever side is required. In particular, Hadamard's lemma states that

$$
\left[\frac{d \phi}{d m}\right]=\frac{d[\phi]}{d m} .
$$

The general assumptions of this paper, following [6], [8], and [9], are that the position vector $\mathbf{x}$ is singular of order two at $C_{t}$, meaning that $\mathbf{h}_{i}, \mathbf{h}^{i}, h^{\alpha \beta}$, and $h_{\alpha \beta}$ are continuous, but that the Christoffel symbols and $\Omega_{\alpha \beta}$ are not in general continuous across $C_{t}$, and that $\phi$ is smooth except at $C_{t}$. In addition we assume of $\phi$ that its limit at $C_{t}$ and the limit of its directional derivative normal to $C_{t}$ (defined in Eq. (4.2)) from each side vanish, that is,

$$
\phi^{-}=\phi^{+}=\frac{d \phi^{-}}{d n}=\frac{d \phi^{+}}{d n}=0
$$

the second and fourth expressions vanishing because of the vanishing of $\phi$ identically on the positive side of $C_{t}$. In consequence, $[\phi]=0$ and all derivatives of $\phi$ on the positive side of $C_{t}$ have vanishing limits at $C_{t}$. This is a simplifying assumption of the present work as compared with [8], [9], and [10]. Since the aim of this work is to find formulas for jumps, terms that will ultimately vanish will contribute nothing to those formulas. So long as they will not again be differentiated, they can be discarded; the indication that some such terms have been discarded will be the use of the special equal sign, $\doteq$, which first appears in Eq. (3.19). Because many terms are products of $\phi$ or derivatives of $\phi$ in directions tangential to the singular surface and geometrical quantities that may themselves have nonzero jumps across $C_{t}$, as mentioned in the previous paragraph, it is important to note the precise effect of the simplifying assumption. In Eq. (1.5), when $A$ is a geometrical coefficient and $B$ is $\phi$ or $d \phi / d n$, the jump in the product will vanish, the vanishing jump in $\phi$ or $d \phi / d n$ respectively eliminating the first and third terms and the vanishing of $\phi$ or $d \phi / d n$ respectively ahead of $C_{t}$ eliminating the second. Then by Hadamard's lemma applied twice, the same thing happens with terms involving tangential directional derivatives of $\phi$ and $d \phi / d n$, e.g., with respect to the direction tangent to the singular curve. No such term will therefore figure in the final results nor in calculations after the specialization indicated by $\doteq$ has been made.

Despite the lack of an assumption of continuity of the Christoffel symbols and $\Omega_{\alpha \beta}$ across $C_{t}$, Cohen and Wang [6] showed that

$$
\left[\left\{\begin{array}{c}
\lambda \\
\alpha \beta
\end{array}\right\}\right] m^{\beta}=0 \text { and }\left[\Omega_{\alpha \beta}\right] m^{\beta}=0 .
$$

It is necessary to recapitulate the relations among partial differentiation with respect to time, which will be represented by the dot accent, and partial and covariant derivatives with respect to surface coordinates. These have been carefully analyzed by Cohen and Wang [6], whose paper should be consulted for details.

2. Kinematics. Much depends upon the velocity vector field $\mathbf{v}$ of the surface $S_{t}$ in euclidean space. The so-called world velocity of $S_{t}$ is then $\boldsymbol{\omega}=\mathbf{v}+\mathbf{e}_{4}$, where $\mathbf{e}_{4}$ is the 
unit tangent vector of the $t$-coordinate curve through each point of space. To represent the directional derivative with respect to $\boldsymbol{\omega}$, we shall use the dot accent. It will be useful to clarify the use of dots. For vectors, dots are tied to the symbol; $\dot{\phi}_{\mid \alpha \beta}$ is $(\dot{\phi})_{\mid \alpha \beta}$ not $\overline{\phi_{\mid \alpha \beta}}$. For coordinates, dots are tied to the coordinate expression as a whole but not including differentiation subscripts; $\dot{\phi}^{\gamma}$ means $\dot{\phi^{\gamma}}$ not the $\mathbf{h}_{\gamma}$ component of $\dot{\phi}$, and $\dot{\phi}_{\mid \lambda}^{\gamma}$ means $\left(\dot{\phi}^{\gamma}\right)_{\mid \lambda} \operatorname{not} \dot{\phi_{\mid \lambda}^{\gamma}}$ and not $(\dot{\phi})_{\mid \lambda}^{\gamma}$, which has no meaning at all. For any tensor field on $S_{t}$, we write

$$
\dot{\phi}=\frac{\partial \phi\left(\theta^{\alpha}, t\right)}{\partial t}=\frac{d \phi}{d \omega} .
$$

In particular, for $\mathbf{x}\left(\theta^{\alpha}, t\right)$ representing position on $S_{t}$, the velocity $\mathbf{v}\left(\theta^{\alpha}, t\right)$ of $S_{t}$ is

$$
\mathbf{v}=\frac{d \mathbf{x}}{d \omega}=\frac{\partial \mathbf{x}\left(\theta^{\alpha}, t\right)}{\partial t}=\dot{\mathbf{x}}
$$

This velocity can be represented relative to the basis of the tangent space, $\mathbf{v}=v^{\lambda} \mathbf{h}_{\lambda}+$ $v^{3} \mathbf{h}_{3}$. It is not assumed continuous across $C_{t}$, but it is assumed to be smooth elsewhere. If $\mathbf{L}$ represents the surface velocity gradient, $\mathbf{v}_{. \alpha} \otimes \mathbf{h}^{\alpha}$, then from (1.2) we have

$$
\begin{aligned}
\mathbf{L} & =L^{\lambda}{ }_{\alpha} \mathbf{h}_{\lambda} \otimes \mathbf{h}^{\alpha}+L^{3}{ }_{\alpha} \mathbf{h}_{3} \otimes \mathbf{h}^{\alpha} \\
& =\left(v_{\mid \alpha}^{\lambda}-\Omega_{\alpha}^{\lambda} v^{3}\right) \mathbf{h}_{\lambda} \otimes \mathbf{h}^{\alpha}+\left(v_{. \alpha}^{3}+\Omega_{\lambda \alpha} v^{\lambda}\right) \mathbf{h}_{3} \otimes \mathbf{h}^{\alpha} .
\end{aligned}
$$

We shall have occasion to expand both $L_{\beta}^{\alpha}$ and $L_{\beta}^{3}$ in terms of the coordinates of $\mathbf{m}$ and n:

$$
L_{\beta}^{\alpha}=L_{m m} m^{\alpha} m_{\beta}+L_{m n} m^{\alpha} n_{\beta}+L_{n m} n^{\alpha} m_{\beta}+L_{n n} n^{\alpha} n_{\beta}
$$

and

$$
L_{\beta}^{3}=L_{m}^{3} m_{\beta}+L_{n}^{3} n_{\beta}
$$

From

$$
\dot{\mathbf{h}}_{\alpha}=\frac{\partial^{2} \mathbf{x}}{\partial t \partial \theta^{\alpha}}=\frac{\partial^{2} \mathbf{x}}{\partial \theta^{\alpha} \partial t}=\mathbf{v}_{, \alpha}
$$

we have

$$
\dot{\mathbf{h}}_{\alpha}=L_{\alpha}^{\lambda} \mathbf{h}_{\lambda}+L_{\alpha}^{3} \mathbf{h}_{3}=\left(v_{\mid \alpha}^{\lambda}-\Omega_{\alpha}^{\lambda} v^{3}\right) \mathbf{h}_{\lambda}+\left(v_{. \alpha}^{3}+\Omega_{\lambda \alpha} v^{\lambda}\right) \mathbf{h}_{3} .
$$

Then by the perpendicularity of $\mathbf{h}_{\alpha}, \mathbf{h}_{3}$,

$$
\dot{\mathbf{h}}_{3}=-L^{3}{ }_{\alpha} \mathbf{h}^{\alpha}=-L^{3 \alpha} \mathbf{h}_{\alpha}=-\left(v^{3 . \alpha}+\Omega_{\lambda}^{\alpha} v^{\lambda}\right) \mathbf{h}_{\alpha} .
$$

If the singular curve $C_{t}$ has an intrinsic speed of propagation $U$ normal to itself across the surface $S_{t}$, then the world velocity of $C_{t}$ can be called $\mathbf{z}=\boldsymbol{\omega}+U \mathbf{n}$. This relationship among the vectors allows (see [6]) the directional derivative with respect to $\boldsymbol{\omega}$ to be written

$$
\dot{\phi}=\frac{d \phi}{d z}-U \frac{d \phi}{d n}
$$

While the connected displacement derivatives with respect to $z$ have their uses, it has been clearly established by the uses made in [2] and [3] of the derivatives with respect 
to oblique trajectories used in [1], that compatibility conditions should be found with respect to such oblique trajectories although it is more trouble. Accordingly, let there be added to $\mathbf{z}$ a tangential velocity, called $\gamma \mathbf{m}$ in [6], to give the tangential velocity $\boldsymbol{\tau}=\mathbf{z}+\gamma \mathbf{m}$. Then

$$
\frac{d}{d \tau}=\frac{d}{d z}+\gamma \frac{d}{d m}
$$

The normal-trajectory derivatives can of course be recovered from the more general expressions simply by letting $\gamma$ vanish. Finally, since both $\mathbf{z}$ and $\boldsymbol{\tau}$ are tangent to the singular surface swept out in space-time by $C_{t}$, there is a version of Hadamard's lemma for directional derivatives with respect to each,

$$
\left[\frac{d \phi}{d z}\right]=\frac{d[\phi]}{d z}=\frac{d \psi}{d z}, \quad\left[\frac{d \phi}{d \tau}\right]=\frac{d[\phi]}{d \tau}=\frac{d \psi}{d \tau} .
$$

When $\phi$ in (2.9) is $\mathbf{h}_{\alpha},(1.2)$ produces

$$
\frac{d \mathbf{h}_{\alpha}}{d z}=\dot{\mathbf{h}}_{\alpha}+U\left\{\begin{array}{c}
\lambda \\
\alpha \beta
\end{array}\right\} n^{\beta} \mathbf{h}_{\lambda}+U \Omega_{\alpha \beta} n^{\beta} \mathbf{h}_{3} .
$$

With (2.7), this is

$$
\frac{d \mathbf{h}_{\alpha}}{d z}=\left(L^{\lambda}{ }_{\alpha}+U\left\{\begin{array}{c}
\lambda \\
\alpha \beta
\end{array}\right\} n^{\beta}\right) \mathbf{h}_{\lambda}+\left(L_{\alpha}^{3}+U \Omega_{\lambda \alpha} n^{\lambda}\right) \mathbf{h}_{3} .
$$

Then by the perpendicularity of $\mathbf{h}_{\alpha}, \mathbf{h}_{3}$,

$$
\frac{d \mathbf{h}_{3}}{d z}=-\left(L_{\alpha}^{3}+U \Omega_{\alpha \beta} n^{\beta}\right) \mathbf{h}^{\alpha}=-\left(L^{3 \alpha}+U \Omega_{\beta}^{\alpha} n^{\beta}\right) \mathbf{h}_{\alpha} .
$$

Both of these formulas are needed later. The continuity of $\mathbf{h}_{\alpha}, \mathbf{h}_{3}$ at $C_{t}$ together with (2.11), implies

$$
\left[L^{\lambda}{ }_{\alpha}+U\left\{\begin{array}{c}
\lambda \\
\alpha \beta
\end{array}\right\} n^{\beta}\right]=0, \quad\left[L^{3}{ }_{\alpha}+U \Omega_{\alpha \beta} n^{\beta}\right]=0
$$

The former of these conditions and (1.8) have the consequence that, when the operator on coordinates,

$$
\frac{D \phi^{\alpha}}{d z}=\frac{d \phi^{\alpha}}{d z}+\phi^{\lambda}\left(L_{\alpha}^{\lambda}+U\left\{\begin{array}{c}
\lambda \\
\alpha \beta
\end{array}\right\} n^{\beta}\right)
$$

is defined, and when the analogous operator

$$
\frac{D \phi^{\alpha}}{d \tau}=\frac{d \phi^{\alpha}}{d \tau}+\phi^{\lambda}\left(L_{\alpha}^{\lambda}+U\left\{\begin{array}{c}
\lambda \\
\alpha \beta
\end{array}\right\} n^{\beta}+\gamma\left\{\begin{array}{c}
\lambda \\
\alpha \beta
\end{array}\right\} m^{\beta}\right)=\frac{D \phi^{\alpha}}{d z}+\gamma \frac{D \phi^{\alpha}}{D m}
$$

is defined, they satisfy versions of Hadamard's lemma,

$$
\left[\frac{D \phi^{\alpha}}{d z}\right]=\frac{D\left[\phi^{\alpha}\right]}{d z}=\frac{D \psi^{\alpha}}{d z}, \quad\left[\frac{D \phi^{\alpha}}{d \tau}\right]=\frac{D\left[\phi^{\alpha}\right]}{d \tau}=\frac{D \psi^{\alpha}}{d \tau} .
$$


Four derivatives derived by Cohen and Wang [6] and misquoted in [8] are needed later:

$$
\begin{aligned}
& \frac{d m_{\lambda}}{d z}=\frac{d U}{d m} n_{\lambda}-L^{\alpha}{ }_{\beta} m_{\alpha} m^{\beta} m_{\lambda}+U\left\{\begin{array}{c}
\alpha \\
\lambda \beta
\end{array}\right\} m_{\alpha} n^{\beta}+\left(L_{\lambda \beta}+L_{\beta \lambda}\right) m^{\beta}, \\
& \frac{d n_{\lambda}}{d z}=-\frac{d U}{d m} m_{\lambda}+L^{\alpha}{ }_{\beta} n_{\alpha} n^{\beta} n_{\lambda}+U\left\{\begin{array}{c}
\alpha \\
\lambda \beta
\end{array}\right\} n_{\alpha} n^{\beta}, \\
& \frac{d m^{\lambda}}{d z}=\frac{d U}{d m} n^{\lambda}-L^{\alpha}{ }_{\beta} m_{\alpha} m^{\beta} m^{\lambda}-U\left\{\begin{array}{c}
\lambda \\
\alpha \beta
\end{array}\right\} m^{\alpha} n^{\beta}, \\
& \frac{d n^{\lambda}}{d z}=-\frac{d U}{d m} m^{\lambda}+L^{\alpha}{ }_{\beta} n_{\alpha} n^{\beta} n^{\lambda}-U\left\{\begin{array}{c}
\lambda \\
\alpha \beta
\end{array}\right\} n^{\alpha} n^{\beta}-\left(L^{\lambda \beta}+L^{\beta \lambda}\right) n_{\beta} .
\end{aligned}
$$

Using the expansion of $L^{\alpha}{ }_{\beta}$, one can write, from [8],

$$
\frac{D m^{\beta}}{d z}=\left(\frac{d U}{d m}+L_{n m}\right) n^{\beta}, \quad \frac{D n^{\beta}}{d z}=-\left(\frac{d U}{d m}+L_{n m}\right) m^{\beta} .
$$

3. Commutation relations. We recall from [6] the definition

$$
\phi_{\mid \alpha \beta}=\phi_{, \alpha \beta}-\phi_{, \gamma}\left\{\begin{array}{c}
\gamma \\
\alpha \beta
\end{array}\right\}=\frac{d^{2} \phi}{d h_{\beta} d h_{\alpha}}-\frac{d \phi}{d h_{\gamma}}\left\{\begin{array}{c}
\gamma \\
\alpha \beta
\end{array}\right\}
$$

and from [9] the further definition

$$
\begin{aligned}
\phi_{\mid \alpha \beta \delta}= & \phi_{, \beta \alpha \delta}-\phi_{, \gamma}\left\{\begin{array}{c}
\gamma \\
\beta \alpha
\end{array}\right\}_{, \delta}-\phi_{, \gamma \delta}\left\{\begin{array}{c}
\gamma \\
\beta \alpha
\end{array}\right\}-\phi_{, \theta} \Omega_{\alpha}^{\theta} \Omega_{\beta \delta} \\
& -\phi_{, \theta} \Omega_{\beta}^{\theta} \Omega_{\alpha \delta}-\phi_{\mid \eta \beta}\left\{\begin{array}{c}
\eta \\
\delta \alpha
\end{array}\right\}-\phi_{\mid \alpha \theta}\left\{\begin{array}{c}
\theta \\
\delta \beta
\end{array}\right\} .
\end{aligned}
$$

Two commutation relations for directional derivatives for fixed time, which will be needed later, can be deduced from expressions for $\phi_{\mid \alpha \beta \delta}$ obtained by iterating

$$
\operatorname{grad} \phi=\frac{d \phi}{d m} \otimes \mathbf{m}+\frac{d \phi}{d n} \otimes \mathbf{n}
$$

in component form,

$$
\frac{d \phi}{d h_{\alpha}}=\frac{d \phi}{d m} m_{\alpha}+\frac{d \phi}{d n} n_{\alpha}
$$

A number of terms that arise can be simplified using the expression

$$
\Omega_{\alpha \beta}=\Omega_{m m} m_{\alpha} m_{\beta}+\Omega_{m n} m_{\alpha} n_{\beta}+\Omega_{n m} n_{\alpha} m_{\beta}+\Omega_{n n} n_{\alpha} n_{\beta}
$$

for the second fundamental form, where $\Omega_{n m}=\Omega_{m n}$ by its symmetry. The expression 
obtained (from [9] based on [6]) is not identical to but is equal to

$$
\begin{aligned}
& \phi_{\mid \alpha \beta \delta}=\left(\frac{d^{3} \phi}{d m^{3}}-\frac{d \eta}{d m} \frac{d \phi}{d n}-3 \eta \frac{d^{2} \phi}{d m d n}-2 \eta^{2} \frac{d \phi}{d m}-2 \Omega_{m m}^{2} \frac{d \phi}{d m}\right. \\
& \left.-2 \Omega_{m n} \Omega_{m m} \frac{d \phi}{d n}\right) m_{\alpha} m_{\beta} m_{\delta} \\
& +\left\{\frac{d^{3} \boldsymbol{\phi}}{d m^{2} d n}+\frac{d \eta}{d m} \frac{d \phi}{d m}+2 \eta \frac{d^{2} \boldsymbol{\phi}}{d m^{2}}-\eta^{2} \frac{d \phi}{d n}-\eta\left(\frac{d^{2} \boldsymbol{\phi}}{d n^{2}}-\mu \frac{d \phi}{d m}\right)-2 \Omega_{m m} \Omega_{m n} \frac{d \phi}{d m}\right. \\
& \left.-\left(\Omega_{m n}^{2}+\Omega_{n n} \Omega_{m m}\right) \frac{d \phi}{d n}\right\}\left(m_{\alpha} m_{\beta} n_{\delta}+m_{\alpha} n_{\beta} m_{\delta}+n_{\alpha} m_{\beta} m_{\delta}\right) \\
& +\left\{\left(\frac{d^{3} \boldsymbol{\phi}}{d m d n^{2}}-\frac{d \mu}{d m} \frac{d \boldsymbol{\phi}}{d m}-\mu \frac{d^{2} \boldsymbol{\phi}}{d m^{2}}\right)+2 \eta \frac{d^{2} \boldsymbol{\phi}}{d m d n}+2 \eta^{2} \frac{d \boldsymbol{\phi}}{d m}\right. \\
& \left.-2 \Omega_{m n}^{2} \frac{d \phi}{d m}-2 \Omega_{n n} \Omega_{m n} \frac{d \phi}{d n}\right\}\left(m_{\alpha} n_{\beta} n_{\delta}+n_{\alpha} m_{\beta} n_{\delta}+n_{\alpha} n_{\beta} m_{\delta}\right) \\
& +\left\{\left(\frac{d^{3} \phi}{d n^{3}}-\frac{d \mu}{d n} \frac{d \phi}{d m}-\mu \frac{d^{2} \phi}{d n d m}\right)-2 \mu \frac{d^{2} \phi}{d m d n}\right. \\
& \left.-2 \mu \eta \frac{d \phi}{d m}-2 \Omega_{m n} \Omega_{n n} \frac{d \phi}{d m}-2 \Omega_{n n}^{2} \frac{d \phi}{d n}\right\} n_{\alpha} n_{\beta} n_{\delta},
\end{aligned}
$$

where $\eta$ is the geodesic curvature of $C_{t}$ and $\mu$ is the geodesic curvature of the orthogonal trajectories on $S_{t}$ of the family of curves $C_{t}$, both defined in terms of covariant directional derivatives by

$$
\frac{D m^{\alpha}}{D m}=m_{\mid \lambda}^{\alpha} m^{\lambda}=\eta n^{\alpha}, \quad \frac{D n^{\alpha}}{D n}=n_{\mid \lambda}^{\alpha} n^{\lambda}=\mu m^{\alpha} .
$$

Because the quantity $\phi_{\mid \alpha \beta \delta}$ is symmetric in the suffixes $\alpha, \beta$, and $\delta$, the coefficients of $m_{\alpha} n_{\beta} n_{\delta}$ and $n_{\alpha} m_{\beta} n_{\delta}$ that actually appear in the computation must be equal to that of $n_{\alpha} n_{\beta} m_{\delta}$, which appears above. That is,

$$
\begin{aligned}
& \frac{d}{d n}\left(\frac{d^{2} \boldsymbol{\phi}}{d m d n}+\eta \frac{d \boldsymbol{\phi}}{d m}\right)-\mu\left(\frac{d^{2} \boldsymbol{\phi}}{d m^{2}}-\eta \frac{d \boldsymbol{\phi}}{d n}\right)+\mu\left(\frac{d^{2} \boldsymbol{\phi}}{d n^{2}}-\mu \frac{d \boldsymbol{\phi}}{d m}\right) \\
& -\left(\Omega_{m n}^{2}+\Omega_{m m} \Omega_{n n}\right) \frac{d \boldsymbol{\phi}}{d m}-2 \Omega_{n n} \Omega_{m n} \frac{d \boldsymbol{\phi}}{d n} \\
& =\frac{d^{3} \boldsymbol{\phi}}{d m d n^{2}}-\frac{d \mu}{d m} \frac{d \phi}{d m}-\mu \frac{d^{2} \boldsymbol{\phi}}{d m^{2}}+2 \eta \frac{d^{2} \boldsymbol{\phi}}{d m d n}+2 \eta^{2} \frac{d \boldsymbol{\phi}}{d m} \\
& \quad-2 \Omega_{m n}^{2} \frac{d \boldsymbol{\phi}}{d m}-2 \Omega_{n n} \Omega_{m n} \frac{d \phi}{d n},
\end{aligned}
$$

which can be written more usefully as

$$
\begin{aligned}
\frac{d}{d n}\left(\frac{d^{2} \boldsymbol{\phi}}{d m d n}+\eta \frac{d \boldsymbol{\phi}}{d m}\right)= & \frac{d^{3} \boldsymbol{\phi}}{d m d n^{2}}+2 \eta \frac{d^{2} \boldsymbol{\phi}}{d m d n}-\mu \frac{d^{2} \boldsymbol{\phi}}{d n^{2}} \\
& +\left(2 \eta^{2}+\mu^{2}+K-\frac{d \mu}{d m}\right) \frac{d \boldsymbol{\phi}}{d m}-\mu \eta \frac{d \boldsymbol{\phi}}{d n}
\end{aligned}
$$

letting

$$
K=\Omega_{m m} \Omega_{n n}-\Omega_{m n}^{2}
$$


be the Gaussian curvature of the surface. Likewise the coefficient of $m_{\alpha} m_{\beta} n_{\delta}$ that actually appears must be equal to that of $m_{\alpha} n_{\beta} m_{\delta}$ and $n_{\alpha} m_{\beta} m_{\delta}$, which appears above;

$$
\begin{aligned}
\frac{d}{d n}( & \left.\frac{d^{2} \boldsymbol{\phi}}{d m^{2}}-\eta \frac{d \phi}{d n}\right)+2 \mu\left(\frac{d^{2} \boldsymbol{\phi}}{d m d n}+\eta \frac{d \boldsymbol{\phi}}{d m}\right) \\
& -2\left(\Omega_{m m} \Omega_{m n} \frac{d \phi}{d m}+\Omega_{m n}^{2} \frac{d \phi}{d n}\right) \\
= & \frac{d^{3} \boldsymbol{\phi}}{d m^{2} d n}+\frac{d \eta}{d m} \frac{d \phi}{d m}+2 \eta \frac{d^{2} \boldsymbol{\phi}}{d m^{2}}-\eta^{2} \frac{d \phi}{d n}-\eta\left(\frac{d^{2} \boldsymbol{\phi}}{d n^{2}}-\mu \frac{d \phi}{d m}\right) \\
& -2 \Omega_{m m} \Omega_{m n} \frac{d \phi}{d m}-\left(\Omega_{m n}^{2}+\Omega_{n n} \Omega_{m m}\right) \frac{d \phi}{d n}
\end{aligned}
$$

which can be written

$$
\begin{aligned}
\frac{d}{d n}\left(\frac{d^{2} \boldsymbol{\phi}}{d m^{2}}-\eta \frac{d \boldsymbol{\phi}}{d n}\right)= & \frac{d^{3} \boldsymbol{\phi}}{d m^{2} d n}+2 \eta \frac{d^{2} \boldsymbol{\phi}}{d m^{2}}-2 \mu \frac{d^{2} \boldsymbol{\phi}}{d m d n}-\eta \frac{d^{2} \boldsymbol{\phi}}{d n^{2}} \\
& +\left(\frac{d \eta}{d m}-\mu \eta\right) \frac{d \boldsymbol{\phi}}{d m}-\left(\eta^{2}+K\right) \frac{d \phi}{d n}
\end{aligned}
$$

A pair of commutation relations involving differentiation with respect to time will be needed later. They can be derived from the equality of partial derivatives,

$$
\frac{\partial^{3} \phi}{\partial t \partial \theta^{\beta} \partial \theta^{\alpha}}=\frac{\partial^{3} \phi}{\partial \theta^{\beta} \partial t \partial \theta^{\alpha}}
$$

and an expression derived in [6],

$$
\overline{\left\{\begin{array}{c}
\gamma \\
\alpha \beta
\end{array}\right\}}=L^{3 \gamma} \Omega_{\alpha \beta}+L_{\alpha \mid \beta}^{\gamma}-L_{\alpha}^{3} \Omega_{\beta}^{\gamma},
$$

expanded in terms of $m_{\beta}$ and $n_{\beta}$ :

$$
\begin{aligned}
\overline{\left\{\begin{array}{c}
\gamma \\
\alpha \beta
\end{array}\right\}=} & \left(\frac{D L^{\gamma} \alpha}{D m}+L^{3 \gamma}\left(\Omega_{m m} m_{\alpha}+\Omega_{n m} n_{\alpha}\right)-L_{\alpha}^{3}\left(\Omega_{m m} m^{\gamma}+\Omega_{n m} n^{\gamma}\right)\right) m_{\beta} \\
& +\left(\frac{D L^{\gamma} \alpha}{D n}+L^{3 \gamma}\left(\Omega_{m n} m_{\alpha}+\Omega_{n n} n_{\alpha}\right)-L_{\alpha}^{3}\left(\Omega_{m n} m^{\gamma}+\Omega_{n n} n^{\gamma}\right)\right) n_{\beta} .
\end{aligned}
$$

We shall need the coefficient of $m_{\beta}$ also expanded in terms of $m_{\alpha}$ and $n_{\alpha}$ :

$$
\begin{aligned}
&\left(\frac{d L_{m m}}{d m} m^{\gamma}+\frac{d L_{n m}}{d m} n^{\gamma}+\eta L_{m m} n^{\gamma}-\eta L_{m n} m^{\gamma}\right.-\eta L_{n m} m^{\gamma}+\eta L_{n n} n^{\gamma} \\
&\left.+L^{3 n} \Omega_{m m} n^{\gamma}-L^{3 m} \Omega_{n m} n^{\gamma}\right) m_{\alpha} \\
&+\left(\frac{d L_{m n}}{d m} m^{\gamma}+\frac{d L_{n n}}{d m} n^{\gamma}+\eta L_{m m} m^{\gamma}+\eta L_{m n} n^{\gamma}+\eta L_{n m} n^{\gamma}-\eta L_{n n} m^{\gamma}\right. \\
&\left.+L^{3 m} \Omega_{n m} m^{\gamma}-L^{3 n} \Omega_{m m} m^{\gamma}\right) n_{\alpha}
\end{aligned}
$$


The left side of $(3.13)$ is

$$
\overline{\phi_{, \alpha \beta}}=\overline{\phi_{\mid \alpha \beta}}+\dot{\phi}_{, \gamma}\left\{\begin{array}{c}
\gamma \\
\alpha \beta
\end{array}\right\}+\phi_{, \gamma} \overline{\left\{\begin{array}{c}
\gamma \\
\alpha \beta
\end{array}\right\}}
$$

and can be expanded using (3.15) and an expansion for $\overline{\phi_{\mid \alpha \beta}}$ available by partial differentiation with respect to $t$ of $\phi_{\mid \alpha \beta}$, used in previous work on geometrical compatibility conditions in [9]. We have (cf. (2.9))

$$
\begin{aligned}
\frac{\cdot}{\phi_{\mid \alpha \beta}}= & \left(\frac{d}{d z}-U \frac{d}{d n}\right)\left\{\left(\frac{d^{2} \phi}{d m^{2}}-\eta \frac{d \phi}{d n}\right) m_{\alpha} m_{\beta}\right. \\
& \left.+\left(\frac{d^{2} \phi}{d m d n}+\eta \frac{d \phi}{d m}\right)\left(m_{\alpha} n_{\beta}+n_{\alpha} m_{\beta}\right)+\left(\frac{d^{2} \phi}{d n^{2}}-\mu \frac{d \phi}{d m}\right) n_{\alpha} n_{\beta}\right\} .
\end{aligned}
$$

This differentiation requires the commutation relations (3.9) and (3.12) and results in the simplified expression:

$$
\begin{aligned}
\frac{\cdot}{\phi_{\mid \alpha \beta}} \doteq U & \eta \frac{d^{2} \phi}{d n^{2}} m_{\alpha} m_{\beta} \\
& +\left(-U \frac{d^{3} \phi}{d m d n^{2}}-\frac{d U}{d m} \frac{d^{2} \phi}{d n^{2}}\right)\left(m_{\alpha} n_{\beta}+n_{\alpha} m_{\beta}\right) \\
& +\left(\frac{d^{3} \phi}{d z d n^{2}}-U \frac{d^{3} \phi}{d n^{3}}+2 L_{n n} \frac{d^{2} \phi}{d n^{2}}\right) n_{\alpha} n_{\beta}
\end{aligned}
$$

The right side of (3.13) can be obtained by partial differentiation of

$$
\begin{aligned}
\dot{\phi}_{, \alpha}=m_{\alpha} & \left\{\frac{d^{2} \boldsymbol{\phi}}{d z d m}-U \frac{d^{2} \boldsymbol{\phi}}{d m d n}+\left(L_{m m}-U \eta\right) \frac{d \boldsymbol{\phi}}{d m}-\frac{d U}{d m} \frac{d \boldsymbol{\phi}}{d n}\right\} \\
+ & n_{\alpha}\left\{\frac{d^{2} \boldsymbol{\phi}}{d z d n}-U \frac{d^{2} \boldsymbol{\phi}}{d n^{2}}+\left(\frac{d U}{d m}+L_{n m}+L_{m n}+U \mu\right) \frac{d \boldsymbol{\phi}}{d m}+L_{n n} \frac{d \boldsymbol{\phi}}{d n}\right\},
\end{aligned}
$$

from [8], where it is Eq. $\left(4.2_{2}\right)$. So, in somewhat simplified form,

$$
\begin{aligned}
\frac{d}{d m} \dot{\boldsymbol{\phi}}_{, \alpha} m_{\beta} & +\frac{d}{d n} \dot{\boldsymbol{\phi}}_{, \alpha} n_{\beta} \\
\doteq & \eta U \frac{d^{2} \boldsymbol{\phi}}{d n^{2}} m_{\alpha} m_{\beta}+\frac{d}{d m}\left\{-U \frac{d^{2} \boldsymbol{\phi}}{d n^{2}}\right\} n_{\alpha} m_{\beta}-\mu U \frac{d^{2} \boldsymbol{\phi}}{d n^{2}} m_{\alpha} n_{\beta} \\
& +\frac{d}{d n}\left\{\frac{d^{2} \phi}{d z d m}-U \frac{d^{2} \boldsymbol{\phi}}{d m d n}+\left(L_{m m}-U \eta\right) \frac{d \phi}{d m}-\frac{d U}{d m} \frac{d \phi}{d n}\right\} m_{\alpha} n_{\beta} \\
& +\frac{d}{d n}\left\{\frac{d^{2} \phi}{d z d n}-U \frac{d^{2} \phi}{d n^{2}}+\left(\frac{d U}{d m}+L_{n m}+L_{m n}+U \mu\right) \frac{d \phi}{d m}+L_{n n} \frac{d \phi}{d n}\right\} n_{\alpha} n_{\beta} \\
& +\dot{\phi}_{, \gamma}\left\{\begin{array}{c}
\gamma \\
\alpha \beta
\end{array}\right\},
\end{aligned}
$$


where the partial derivatives of $m_{\alpha}$ and $n_{\alpha}$ have been expressed in terms of their covariant derivatives, which give rise to terms containing Christoffel symbols, collected above into the final term.

When the above two expressions for the equal partial derivatives, (3.21) and (3.17) elaborated by (3.19), are equated, and the term common to both is dropped, the result as a whole is of no particular interest, but the equality of the coefficients of $n_{\alpha} m_{\beta}$ gives one commutation relation needed later and the equality of the coefficients of $n_{\beta}$ is the other commutation relation sought. (It could be separated into two relations, but they will be needed together.)

The first equality simplifies to

$$
\frac{d^{3} \phi}{d m d z d n} \doteq \frac{d^{3} \phi}{d z d m d n}
$$

where (3.16) has been used. The second equality (that of the coefficients of $n_{\beta}$ ) simplifies to

$$
\begin{aligned}
& \frac{d}{d n}\left\{\frac{d^{2} \boldsymbol{\phi}}{d z d m}-U \frac{d^{2} \boldsymbol{\phi}}{d m d n}+\left(L_{m m}-U \eta\right) \frac{d \boldsymbol{\phi}}{d m}-\frac{d U}{d m} \frac{d \phi}{d n}\right\} m_{\alpha} \\
& \quad+\frac{d}{d n}\left\{\frac{d^{2} \boldsymbol{\phi}}{d z d n}-U \frac{d^{2} \boldsymbol{\phi}}{d n^{2}}+\left(\frac{d U}{d m}+L_{n m}+L_{m n}+U \mu\right) \frac{d \boldsymbol{\phi}}{d m}+L_{n n} \frac{d \phi}{d n}\right\} n_{\alpha} \\
& \doteq\left.\left(\mu U-\frac{d U}{d m}\right) \frac{d^{2} \boldsymbol{\phi}}{d n^{2}}-U \frac{d^{3} \boldsymbol{\phi}}{d m d n^{2}}\right\} m_{\alpha} \\
& \quad+\left\{\frac{d^{3} \boldsymbol{\phi}}{d z d n^{2}}-U \frac{d^{3} \boldsymbol{\phi}}{d n^{3}}+2 L_{n n} \frac{d^{2} \boldsymbol{\phi}}{d n^{2}}\right\} n_{\alpha},
\end{aligned}
$$

where (3.15) has been used.

A commutation relation in coordinates that is needed below can be derived by comparison of two equal but different expressions, the $n_{\lambda}$ terms in $\dot{\phi}_{. \lambda}$ from (4.3) and (4.2) in [8],

$$
\frac{d}{d n}\left(\frac{d \phi}{d z}-U \frac{d \phi}{d n}\right)=\frac{d^{2} \phi}{d z d n}-U \frac{d^{2} \phi}{d n^{2}}+\left(\frac{d U}{d m}+L_{n m}+L_{m n}+U \mu\right) \frac{d \phi}{d m}+L_{n n} \frac{d \phi}{d n}
$$

Equating the coefficients of $\mathbf{h}_{\alpha}$ and the $\mathbf{h}_{3}$ respectively gives

$$
\begin{aligned}
\frac{D^{2} \phi^{\alpha}}{D n d z} & -\phi^{\gamma}\left(L_{\gamma}^{3}+U \Omega_{\gamma \lambda} n^{\lambda}\right) \Omega_{\delta}^{\alpha} n^{\delta}-\left(U \mu+\frac{d U}{d m}+L_{m n}+L_{n m}\right) \frac{D \phi^{\alpha}}{D m}-\left(\frac{d U}{d n}+L_{n n}\right) \frac{D \phi^{\alpha}}{D n} \\
& =\frac{D}{d z} \frac{D \phi^{\alpha}}{D n}-\phi^{\delta} \Omega_{\delta \beta} n^{\beta}\left(L^{3 \alpha}+U \Omega_{\gamma}^{\alpha} n^{\gamma}\right)
\end{aligned}
$$

and

$$
\begin{aligned}
\frac{d^{2} \phi^{3}}{d n d z} & -\phi^{3}\left(L^{3 \alpha}+U \Omega_{\lambda}^{\alpha} n^{\lambda}\right) \Omega_{\alpha \delta} n^{\delta}-\left(U \mu+\frac{d U}{d m}+L_{m n}+L_{n m}\right) \frac{d \phi^{3}}{d m}-\left(\frac{d U}{d n}+L_{n n}\right) \frac{d \phi^{3}}{d n} \\
& =\frac{d^{2} \phi^{3}}{d z d n}-\phi^{3} \Omega_{\beta}^{\alpha} n^{\beta}\left(L_{\alpha}^{3}+U \Omega_{\alpha \gamma} n^{\gamma}\right),
\end{aligned}
$$

or more usefully

$$
\frac{D^{2} \phi^{\alpha}}{D n d z} \doteq \frac{D^{2} \phi^{\alpha}}{d z D n} \quad \text { and } \quad \frac{d^{2} \phi^{3}}{d n d z} \doteq \frac{d^{2} \phi^{3}}{d z d n}
$$


4. Directional derivatives. Since the goal is coordinate expressions, it is necessary to introduce coordinates for quantities other than $\phi=\phi^{\alpha} \mathbf{h}_{\alpha}+\phi^{3} \mathbf{h}_{3}, \phi, \beta, \mathbf{L}, \mathbf{m}, \mathbf{n}$, and $\mathbf{v}$. The first directional derivatives of $\phi$ are

$$
\begin{aligned}
\frac{d \phi}{d m} & =\left(\frac{D \phi^{\alpha}}{D m}-\phi^{3} \Omega_{\beta}^{\alpha} m^{\beta}\right) \mathbf{h}_{\alpha}+\left(\frac{d \phi^{3}}{d m}+\phi^{\alpha} \Omega_{\alpha \beta} m^{\beta}\right) \mathbf{h}_{3}, \\
\frac{d \phi}{d n} & =\left(\frac{D \phi^{\alpha}}{D n}-\phi^{3} \Omega_{\beta}^{\alpha} n^{\beta}\right) \mathbf{h}_{\alpha}+\left(\frac{d \phi^{3}}{d n}+\phi^{\alpha} \Omega_{\alpha \beta} n^{\beta}\right) \mathbf{h}_{3} .
\end{aligned}
$$

The jump in the normal directional derivative of $\phi$ is defined to be

$$
\tilde{\chi}=\left[\frac{d \phi}{d n}\right]=\left(\left[\frac{D \phi^{\alpha}}{D n}\right]-\left[\phi^{3} \Omega_{\beta}^{\alpha} n^{\beta}\right]\right) \mathbf{h}_{\alpha}+\left(\left[\frac{d \phi^{3}}{d n}\right]+\left[\phi^{\alpha} \Omega_{\alpha \beta} n^{\beta}\right]\right) \mathbf{h}_{3} .
$$

If we let

$$
\psi^{\alpha}=\left[\phi^{\alpha}\right], \quad \psi^{3}=\left[\phi^{3}\right], \quad \tilde{\psi}^{\alpha}=\left[\frac{D \phi^{\alpha}}{D n}\right], \quad \tilde{\psi}^{3}=\left[\frac{d \phi^{3}}{d n}\right],
$$

we can note that, while $\tilde{\psi}^{\alpha}, \tilde{\psi}^{3}$, are not the coordinates of $\tilde{\chi}$, hence the use of different letters, they are equal to those coordinates on account of the simplifying assumption on $\phi$ without that on $d \phi / d n$. On account of this much simplification, the first-order compatibility conditions (from [6]) become just

$$
\left[\phi_{\mid \beta}^{\alpha}\right] \doteq \tilde{\psi}^{\alpha} n_{\beta}, \quad\left[\dot{\phi}^{\alpha}\right] \doteq-U \tilde{\psi}^{\alpha}, \quad\left[\phi_{, \beta}^{3}\right] \doteq \tilde{\psi}^{3} n_{\beta}, \quad\left[\dot{\phi}^{3}\right] \doteq-U \psi^{3} .
$$

The second-order directional derivatives are:

$$
\begin{aligned}
& \frac{d^{2} \phi}{d m^{2}}=\left(\frac{D^{2} \phi^{\lambda}}{D m^{2}}-\phi^{\gamma} \Omega_{\gamma \beta} \Omega_{\delta}^{\lambda} m^{\beta} m^{\delta}-2 \frac{d \phi^{3}}{d m} \Omega_{\beta}^{\lambda} m^{\beta}-\phi^{3} \frac{D \Omega_{\beta}^{\lambda}}{D m} m^{\beta}-\eta \phi^{3} \Omega_{\beta}^{\lambda} n^{\beta}\right) \mathbf{h}_{\lambda} \\
&+\left(2 \frac{D \phi^{\alpha}}{D m} \Omega_{\alpha \beta} m^{\beta}+\phi^{\alpha} \frac{D \Omega_{\alpha \beta}}{D m} m^{\beta}+\eta \phi^{\alpha} \Omega_{\alpha \beta} n^{\beta}+\frac{d^{2} \phi^{3}}{d m^{2}}-\phi^{3} \Omega_{\beta}^{\alpha} \Omega_{\alpha \delta} m^{\beta} m^{\delta}\right) \mathbf{h}_{3}, \\
& \frac{d^{2} \phi}{d n^{2}}=\left(\frac{D^{2} \phi^{\lambda}}{D n^{2}}-\phi^{\gamma} \Omega_{\gamma \beta} \Omega_{\delta}^{\lambda} n^{\beta} n^{\delta}-2 \frac{d \phi^{3}}{d n} \Omega_{\beta}^{\lambda} n^{\beta}-\phi^{3} \frac{D \Omega_{\beta}^{\lambda}}{D n} n^{\beta}-\mu \phi^{3} \Omega_{\beta}^{\lambda} m^{\beta}\right) \mathbf{h}_{\lambda} \\
&+\left(2 \frac{D \phi^{\alpha}}{D n} \Omega_{\alpha \beta} n^{\beta}+\phi^{\alpha} \frac{D \Omega_{\alpha \beta}}{D n} n^{\beta}+\mu \phi^{\alpha} \Omega_{\alpha \beta} m^{\beta}+\frac{d^{2} \phi^{3}}{d n^{2}}-\phi^{3} \Omega_{\beta}^{\alpha} \Omega_{\alpha \delta} n^{\beta} n^{\delta}\right) \mathbf{h}_{3}, \\
& \frac{d^{2} \phi}{d m d n}=\left(\frac{D^{2} \phi^{\lambda}}{D m^{2} D}-\phi^{\gamma} \Omega_{\gamma \beta} \Omega_{\delta}^{\lambda} m^{\delta} n^{\beta}-\frac{d \phi^{3}}{d m} \Omega_{\beta}^{\lambda} n^{\beta}-\frac{d \phi^{3}}{d n} \Omega_{\beta}^{\gamma} m^{\beta}\right. \\
&+\left(\frac{D \phi^{\alpha}}{D n} \Omega_{\alpha \beta} m^{\beta}+\frac{D \phi_{\beta}^{\lambda}}{D m} n^{\beta}+\eta \phi^{3} \Omega_{\beta}^{\lambda} m^{\beta}\right) \mathbf{h}_{\lambda} \\
& \\
& \quad \Omega_{\alpha \beta} n^{\beta}+\phi^{\alpha} \frac{D \Omega_{\alpha \beta}}{D m} n^{\beta}-\eta \phi^{\alpha} \Omega_{\alpha \beta} m^{\beta} \\
&\left.+\frac{d^{2} \phi^{3}}{d m d n}-\phi^{3} \Omega_{\eta}^{\alpha} \Omega_{\alpha \beta} m^{\beta} n^{\eta}\right) \mathbf{h}_{3} .
\end{aligned}
$$


Again let

$$
\tilde{\widetilde{\chi}}=\left[\frac{d^{2} \phi}{d n^{2}}-\mu \frac{d \phi}{d m}\right]=\left[\phi_{\mid \alpha \beta} n^{\alpha} n^{\beta}\right] \doteq\left[\frac{d^{2} \phi}{d n^{2}}\right]
$$

and also, in coordinates,

$$
\tilde{\tilde{\psi}}^{\lambda}=\left[\frac{D^{2} \phi^{\lambda}}{D n^{2}}-\mu \frac{D \phi^{\lambda}}{D m}\right] \doteq\left[\frac{D^{2} \phi^{\lambda}}{D n^{2}}\right], \quad \tilde{\tilde{\psi}}^{3}=\left[\frac{d^{2} \phi^{3}}{d n^{2}}-\mu \frac{d \phi^{3}}{d m}\right] \doteq\left[\frac{d^{2} \phi^{3}}{d n^{2}}\right] .
$$

Again, these are not in general the coordinates of $\widetilde{\widetilde{\chi}}$;

$$
\begin{aligned}
\tilde{\widetilde{\chi}}= & \left(\tilde{\tilde{\psi}}^{\lambda}-\left[\phi^{\sigma} \Omega_{\sigma \beta} \Omega_{\gamma}^{\lambda} n^{\beta} n^{\gamma}\right]-2\left[\frac{d \phi^{3}}{d n} \Omega_{\gamma}^{\lambda} n^{\gamma}\right]-\left[\phi^{3} \frac{D \Omega_{\gamma}^{\lambda}}{D n} n^{\gamma}\right]\right) \mathbf{h}_{\lambda} \\
& +\left(2\left[\frac{D \phi^{\alpha}}{D n} \Omega_{\alpha \beta} n^{\beta}\right]+\left[\phi^{\alpha} \frac{D \Omega_{\alpha \beta}}{D n} n^{\beta}\right]+\tilde{\psi}^{3}-\left[\phi^{3} \Omega_{\gamma}^{\alpha} \Omega_{\alpha \beta} n^{\gamma} n^{\beta}\right]\right) \mathbf{h}_{3} .
\end{aligned}
$$

Under the present simplifying assumptions on $\phi$ and $d \phi / d n$, the second-order compatibility conditions are as follows: first the geometric,

$$
\left[\phi_{\mid \alpha \beta}^{\lambda}\right] \doteq \tilde{\tilde{\psi}}^{\lambda} n_{\alpha} n_{\beta} \quad \text { and } \quad\left[\phi_{\mid \alpha \beta}^{3}\right] \doteq \tilde{\tilde{\psi}}^{3} n_{\alpha} n_{\beta}
$$

then the kinematic,

$$
\begin{aligned}
& {\left[\dot{\phi}_{\mid \lambda}^{\alpha}\right] \doteq-U \tilde{\tilde{\psi}}^{\alpha} n_{\lambda} \quad \text { and } \quad\left[\dot{\phi}_{, \lambda}^{3}\right] \doteq-U \tilde{\tilde{\psi}}^{3} n_{\lambda}} \\
& {\left[\ddot{\phi}^{\alpha}\right] \doteq U^{2} \tilde{\tilde{\psi}}^{\alpha} \quad \text { and } \quad\left[\ddot{\phi}^{3}\right] \doteq U^{2} \tilde{\tilde{\psi}}^{3}}
\end{aligned}
$$

The formulas (4.10) were obtained by equating expressions known to be equal in the limit at $C_{t}$ and then taking jumps in those equations. Many terms do not vanish until jumps are taken in them, as can be seen in [8]. It will simplify further work substantially if terms whose jumps will vanish are neglected because when jumps are taken-as they will be-their jumps will vanish even when multiplied by some geometrical quantity whose jump does not vanish. It is therefore as though the above equations had come from the following statements, which are not themselves true but which lead to true statements when jumps are taken. They are needed in what follows:

$$
\begin{gathered}
\dot{\phi}_{\mid \lambda}^{\alpha} \doteq-U \frac{D^{2} \phi^{\alpha}}{D n^{2}} n_{\lambda} \quad \text { and } \quad \dot{\phi}_{, \lambda}^{3} \doteq-U \frac{d^{2} \phi^{3}}{d n^{2}} n_{\lambda} \\
\ddot{\phi}^{\alpha} \doteq U^{2} \frac{D^{2} \phi^{\alpha}}{D n^{2}} \quad \text { and } \quad \ddot{\phi}^{3} \doteq U^{2} \frac{d^{2} \phi^{3}}{d n^{2}}
\end{gathered}
$$

Similar statements from which the first-order kinematic compatibility conditions (4.4) can be derived in their fully simplified form are just the vanishing of the four quantities involved,

$$
\phi_{\mid \beta}^{\alpha} \doteq \phi_{, \beta}^{3} \doteq \dot{\phi}^{\alpha} \doteq \dot{\phi}^{3} \doteq 0
$$


The third-order directional derivatives not involving time are obtained by differentiating the expressions (4.5):

$$
\begin{aligned}
\frac{d^{3} \phi}{d m^{3}}=( & \frac{D^{3} \phi^{\alpha}}{D m^{3}}-3 \frac{D \phi^{\delta}}{D m} \Omega_{\delta \eta} m^{\eta} \Omega_{\beta}^{\alpha} m^{\beta}-2 \phi^{\delta} \frac{D \Omega_{\delta \eta} m^{\eta}}{D m} \Omega_{\beta}^{\alpha} m^{\beta}-\phi^{\gamma} \Omega_{\gamma \beta} m^{\beta} \frac{D \Omega_{\delta}^{\alpha} m^{\delta}}{D m} \\
& \left.-3 \frac{d^{2} \phi^{3}}{d m^{2}} \Omega_{\beta}^{\alpha} m^{\beta}-3 \frac{d \phi^{3}}{d m} \frac{D \Omega_{\beta}^{\alpha} m^{\beta}}{D m}+\phi^{3} \Omega_{\eta}^{\gamma} \Omega_{\gamma \delta} m^{\eta} m^{\delta} \Omega_{\beta}^{\alpha} m^{\beta}-\phi^{3} \frac{D^{2} \Omega_{\beta}^{\alpha} m^{\beta}}{D m^{2}}\right) \mathbf{h}_{\alpha} \\
& +\left(3 \frac{D^{2} \phi^{\alpha}}{D m^{2}} \Omega_{\alpha \beta} m^{\beta}+3 \frac{D \phi^{\alpha}}{D m} \frac{D \Omega_{\alpha \beta} m^{\beta}}{D m}-\phi^{\gamma} \Omega_{\gamma \eta} \Omega_{\delta}^{\alpha} m^{\eta} m^{\delta} \Omega_{\alpha \beta} m^{\beta}\right. \\
& \left.+\phi^{\alpha} \frac{D^{2} \Omega_{\alpha \beta} m^{\beta}}{D m^{2}}+\frac{d^{3} \phi^{3}}{d m^{3}}-3 \frac{d \phi^{3}}{d m} \Omega_{\eta}^{\alpha} m^{\eta} \Omega_{\alpha \beta} m^{\beta}-3 \phi^{3} \frac{D \Omega_{\eta}^{\alpha} m^{\eta}}{D m} \Omega_{\alpha \beta} m^{\beta}\right) \mathbf{h}_{3}
\end{aligned}
$$

$$
\begin{aligned}
\frac{d^{3} \phi}{d m^{2} d n}= & \left(\frac{D^{3} \phi^{\alpha}}{D m^{2} D n}-\frac{D \phi^{\gamma} \Omega_{\gamma \beta} n^{\beta} \Omega_{\delta}^{\alpha} m^{\delta}}{D m}-\frac{D^{2} \phi^{3} \Omega_{\beta}^{\alpha} n^{\beta}}{D m^{2}}-2 \frac{d^{2} \phi^{3}}{d m d n} \Omega_{\beta}^{\alpha} m^{\beta}\right. \\
& -\frac{d \phi^{3}}{d n} \frac{D \Omega_{\beta}^{\alpha} m^{\beta}}{D m}-\frac{D \phi^{\delta}}{D n} \Omega_{\delta \eta} m^{\eta} \Omega_{\beta}^{\alpha} m^{\beta}-\frac{D \phi^{\delta} \Omega_{\delta \eta} n^{\eta}}{D m} \Omega_{\beta}^{\alpha} m^{\beta} \\
& \left.+\phi^{3} \Omega_{\eta}^{\delta} n^{\eta} \Omega_{\delta \gamma} m^{\gamma} \Omega_{\beta}^{\alpha} m^{\beta}\right) \mathbf{h}_{\alpha} \\
& +\left(2 \frac{D^{2} \phi^{\alpha}}{D m D n} \Omega_{\alpha \beta} m^{\beta}+\frac{D \phi^{\alpha}}{D n} \frac{D \Omega_{\alpha \beta} m^{\beta}}{D m}+\frac{D^{2} \phi^{\alpha} \Omega_{\alpha \beta} n^{\beta}}{D m^{2}}\right. \\
& -\phi^{\gamma} \Omega_{\gamma \eta} n^{\eta} \Omega_{\delta}^{\alpha} m^{\delta} \Omega_{\alpha \beta} m^{\beta}+\frac{d^{3} \phi^{3}}{d m^{2} d n}-\frac{d \phi^{3} \Omega_{\eta}^{\alpha} n^{\eta} \Omega_{\alpha \beta} m^{\beta}}{d m} \\
& \left.-\frac{d \phi^{3} \Omega_{\eta}^{\alpha} n^{\eta}}{d m} \Omega_{\alpha \beta} m^{\beta}-\frac{d \phi^{3}}{d n} \Omega_{\eta}^{\alpha} m^{\eta} \Omega_{\alpha \beta} m^{\beta}\right) \mathbf{h}_{3},
\end{aligned}
$$

$$
\begin{aligned}
\frac{d^{3} \phi}{d m d n^{2}}= & \left(\frac{D^{3} \phi^{\alpha}}{D m D n^{2}}-\frac{D \phi^{\lambda}}{D m} \Omega_{\lambda \beta} \Omega_{\gamma}^{\alpha} n^{\beta} n^{\gamma}-\phi^{\lambda} \frac{D \Omega_{\lambda \beta} \Omega_{\gamma}^{\alpha} n^{\beta} n^{\gamma}}{D m}-2 \frac{d^{2} \phi^{3}}{d m d n} \Omega_{\gamma}^{\alpha} n^{\gamma}\right. \\
& -2 \frac{d \phi^{3}}{d n} \frac{D \Omega_{\gamma}^{\alpha} n^{\gamma}}{D m}-\frac{d \phi^{3}}{d m} \frac{D \Omega_{\gamma}^{\alpha} n^{\gamma}}{D n}-\phi^{3} \frac{D^{2} \Omega_{\gamma}^{\alpha} n^{\gamma}}{D m D n}-\frac{d^{2} \phi^{3}}{d n^{2}} \Omega_{\beta}^{\alpha} m^{\beta} \\
& \left.+\phi^{3} \Omega_{\gamma}^{\delta} \Omega_{\delta \eta} n^{\gamma} n^{\eta} \Omega_{\beta}^{\alpha} m^{\beta}-2 \frac{D \phi^{\delta}}{D n} \Omega_{\delta \eta} n^{\eta} \Omega_{\beta}^{\alpha} m^{\beta}-\phi^{\delta} \frac{D \Omega_{\delta \eta} n^{\eta}}{D n} \Omega_{\beta}^{\alpha} m^{\beta}\right) \mathbf{h}_{\alpha} \\
& +\left(\frac{D^{2} \phi^{\alpha}}{D n^{2}} \Omega_{\alpha \beta} m^{\beta}-\phi^{\lambda} \Omega_{\lambda \eta} \Omega_{\gamma}^{\alpha} n^{\eta} n^{\gamma} \Omega_{\alpha \beta} m^{\beta}-2 \frac{d \phi^{3}}{d n} \Omega_{\gamma}^{\alpha} n^{\gamma} \Omega_{\alpha \beta} m^{\beta}\right. \\
& -\phi^{3} \frac{D \Omega_{\gamma}^{\alpha} n^{\gamma}}{D n} \Omega_{\alpha \beta} m^{\beta}+\frac{d^{3} \phi^{3}}{d m d n^{2}}-\frac{d \phi^{3}}{d m} \Omega_{\gamma}^{\alpha} \Omega_{\alpha \beta} n^{\gamma} n^{\beta}-\phi^{3} \frac{d \Omega_{\gamma}^{\alpha} \Omega_{\alpha \beta} n^{\gamma} n^{\beta}}{d m} \\
& \left.+2 \frac{D^{2} \phi^{\alpha}}{D m D n} \Omega_{\alpha \beta} n^{\beta}+2 \frac{D \phi^{\alpha}}{D n} \frac{D \Omega_{\alpha \beta} n^{\beta}}{D m}+\frac{D \phi^{\alpha}}{D m} \frac{D \Omega_{\alpha \beta} n^{\beta}}{D n}+\phi^{\alpha} \frac{D^{2} \Omega_{\alpha \beta} n^{\beta}}{D m D n}\right) \mathbf{h}_{3},
\end{aligned}
$$




$$
\begin{aligned}
\frac{d^{3} \phi}{d n^{3}}= & \left(\frac{D^{3} \phi^{\alpha}}{D n^{3}}-\frac{D \phi^{\lambda} \Omega_{\lambda \beta} \Omega_{\gamma}^{\alpha} n^{\beta} n^{\gamma}}{D n}-2 \frac{D \phi^{\gamma}}{D n} \Omega_{\gamma \delta} n^{\delta} \Omega_{\beta}^{\alpha} n^{\beta}-\phi^{\gamma} \frac{D \Omega_{\gamma \delta} n^{\delta}}{D n} \Omega_{\beta}^{\alpha} n^{\beta}\right. \\
& \left.-3 \frac{d^{2} \phi^{3}}{d n^{2}} \Omega_{\gamma}^{\alpha} n^{\gamma}-3 \frac{d \phi^{3}}{d n} \frac{D \Omega_{\gamma}^{\alpha} n^{\gamma}}{D n}-\phi^{3} \frac{D^{2} \Omega_{\gamma}^{\alpha} n^{\gamma}}{D n^{2}}+\phi^{3} \Omega_{\gamma}^{\eta} \Omega_{\eta \delta} n^{\gamma} n^{\delta} \Omega_{\beta}^{\alpha} n^{\beta}\right) \mathbf{h}_{\alpha} \\
& +\left(3 \frac{D^{2} \phi^{\alpha}}{D n^{2}} \Omega_{\alpha \beta} n^{\beta}+3 \frac{D \phi^{\alpha}}{D n} \frac{D \Omega_{\alpha \beta} n^{\beta}}{D n}+\phi^{\alpha} \frac{D^{2} \Omega_{\alpha \beta} n^{\beta}}{D n^{2}}-\phi^{\lambda} \Omega_{\lambda \eta} \Omega_{\gamma}^{\alpha} n^{\eta} n^{\gamma} \Omega_{\alpha \beta} n^{\beta}\right. \\
& \left.+\frac{d^{3} \phi^{3}}{d n^{3}}-\frac{d \phi^{3} \Omega_{\gamma}^{\alpha} \Omega_{\alpha \beta} n^{\gamma} n^{\beta}}{d n}-2 \frac{d \phi^{3}}{d n} \Omega_{\gamma}^{\alpha} n^{\gamma} \Omega_{\alpha \beta} n^{\beta}-\phi^{3} \frac{D \Omega_{\gamma}^{\alpha} n^{\gamma}}{D n} \Omega_{\alpha \beta} n^{\beta}\right) \mathbf{h}_{3} .
\end{aligned}
$$

Using the notation $\tilde{\tilde{\tilde{\chi}}}$ for $\left[\phi_{\mid \alpha \beta \delta} n^{\alpha} n^{\beta} n^{\delta}\right]$, which is available as the coefficient of $n_{\alpha} n_{\beta} n_{\delta}$ in the expression (3.6) for $\phi_{\mid \alpha \beta \delta}$, we also need

$$
\begin{aligned}
\tilde{\tilde{\tilde{\psi}}}^{\alpha} & =\left[\frac{D^{3} \phi^{\alpha}}{D n^{3}}-\frac{d \mu}{d n} \frac{D \phi^{\alpha}}{D m}-3 \mu \frac{D^{2} \phi^{\alpha}}{D m D n}-3 \mu \eta \frac{D \phi^{\alpha}}{D m}+\mu^{2} \frac{D \phi^{\alpha}}{D n}+2 \mu \phi^{\nu} R_{\nu \gamma \beta}^{\alpha} m^{\gamma} n^{\beta}\right] \\
& \doteq\left[\frac{D^{3} \phi^{\alpha}}{D n^{3}}\right]
\end{aligned}
$$

and

$$
\tilde{\tilde{\psi}}^{3}=\left[\frac{d^{3} \phi^{3}}{d n^{3}}-\frac{d \mu}{d n} \frac{d \phi^{3}}{d m}-3 \mu \frac{d^{2} \phi^{3}}{d m d n}-3 \mu \eta \frac{d \phi^{3}}{d m}+\mu^{2} \frac{d \phi^{3}}{d n}\right] \doteq\left[\frac{d^{3} \phi^{3}}{d n^{3}}\right]
$$

With the notation needed all in place, the third-order geometric conditions of [9] under the simplifying assumptions of this work can now be written out:

$$
\begin{aligned}
\phi_{\mid \alpha \beta \delta}^{\lambda} \doteq- & \eta \tilde{\tilde{\psi}}^{\lambda}\left(m_{\alpha} m_{\beta} n_{\delta}+m_{\alpha} n_{\beta} m_{\delta}+n_{\alpha} m_{\beta} m_{\delta}\right) \\
& +\frac{D \tilde{\tilde{\psi}}^{\lambda}}{D m}\left(m_{\alpha} n_{\beta} n_{\delta}+n_{\alpha} m_{\beta} n_{\delta}+n_{\alpha} n_{\beta} m_{\delta}\right) \\
& +\tilde{\tilde{\tilde{\psi}}}^{\lambda} n_{\alpha} n_{\beta} n_{\delta},
\end{aligned}
$$

and

$$
\begin{aligned}
\phi_{\mid \alpha \beta \delta}^{3} \doteq- & \eta \tilde{\tilde{\psi}}^{3}\left(m_{\alpha} m_{\beta} n_{\delta}+m_{\alpha} n_{\beta} m_{\delta}+n_{\alpha} m_{\beta} m_{\delta}\right) \\
+ & \frac{d \tilde{\tilde{\psi}}^{3}}{d m}\left(m_{\alpha} n_{\beta} n_{\delta}+n_{\alpha} m_{\beta} n_{\delta}+n_{\alpha} n_{\beta} m_{\delta}\right) \\
& \stackrel{\tilde{\tilde{\psi}}^{3} n_{\alpha} n_{\beta} n_{\delta} .}{ }
\end{aligned}
$$

Directional derivatives involving time will be needed. From (4.5), (4.17), and (4.18) in 
[8] come:

$$
\begin{aligned}
& \frac{d^{2} \boldsymbol{\phi}}{d z d m}=\left(\frac{D^{2} \phi^{\alpha}}{d z D m}-\phi^{\gamma} \Omega_{\gamma \beta} m^{\beta} L^{3 \alpha}-U \phi^{\gamma} \Omega_{\gamma \beta} m^{\beta} \Omega_{\delta}^{\alpha} n^{\delta}\right. \\
& -\frac{d \phi^{3}}{d z} \Omega_{\beta}^{\alpha} m^{\beta}-\phi^{3} \frac{D \Omega_{\beta}^{\alpha}}{d z} m^{\beta}-\frac{d U}{d m} \phi^{3} \Omega_{\beta}^{\alpha} n^{\beta} \\
& \left.-L_{n m} \phi^{3} \Omega_{\beta}^{\alpha} n^{\beta}-\frac{d \phi^{3}}{d m} L^{3 \alpha}-U \frac{d \phi^{3}}{d m} \Omega_{\gamma}^{\alpha} n^{\gamma}\right) \mathbf{h}_{\alpha} \\
& +\left(\frac{d^{2} \phi^{3}}{d z d m}-\phi^{3} \Omega_{\beta}^{\alpha} m^{\beta} L_{\alpha}^{3}-U \phi^{3} \Omega_{\beta}^{\alpha} m^{\beta} \Omega_{\alpha \gamma} n^{\gamma}\right. \\
& +\frac{D \phi^{\alpha}}{d z} \Omega_{\alpha \beta} m^{\beta}+\phi^{\alpha} \frac{D \Omega_{\alpha \beta}}{d z} m^{\beta}+\frac{d U}{d m} \phi^{\alpha} \Omega_{\alpha \beta} n^{\beta} \\
& \left.+L_{n m} \phi^{\alpha} \Omega_{\alpha \beta} n^{\beta}+\frac{D \phi^{\alpha}}{D m} L_{\alpha}^{3}+U \frac{D \phi^{\alpha}}{D m} \Omega_{\alpha \delta} n^{\delta}\right) \mathbf{h}_{3} \\
& \frac{d^{2} \phi}{d z d n}=\left(\frac{D^{2} \phi^{\alpha}}{d z D n}-\phi^{\gamma} \Omega_{\gamma \beta} n^{\beta} L^{3 \alpha}-U \phi^{\gamma} \Omega_{\gamma \beta} n^{\beta} \Omega_{\delta}^{\alpha} n^{\delta}-\frac{d \phi^{3}}{d z} \Omega_{\beta}^{\alpha} n^{\beta}-\phi^{3} \frac{D \Omega_{\beta}^{\alpha}}{d z} n^{\beta}\right. \\
& \left.+\frac{d U}{d m} \phi^{3} \Omega_{\beta}^{\alpha} m^{\beta}+L_{n m} \phi^{3} \Omega_{\beta}^{\alpha} m^{\beta}-\frac{d \phi^{3}}{d n} L^{3 \alpha}-U \frac{d \phi^{3}}{d n} \Omega_{\gamma}^{\alpha} n^{\gamma}\right) \mathbf{h}_{\alpha} \\
& +\left(\frac{d^{2} \phi^{3}}{d z d n}-\phi^{3} \Omega_{\beta}^{\alpha} n^{\beta} L_{\alpha}^{3}-U \phi^{3} \Omega_{\beta}^{\alpha} n^{\beta} \Omega_{\alpha \gamma} n^{\gamma}+\frac{D \phi^{\gamma} \Omega_{\gamma \beta}}{d z} n^{\beta}\right. \\
& \left.-\frac{d U}{d m} \phi^{\gamma} \Omega_{\gamma \beta} m^{\beta}-L_{n m} \phi^{\gamma} \Omega_{\gamma \beta} m^{\beta}+\frac{D \phi^{\alpha}}{D n} L_{\alpha}^{3}+U \frac{D \phi^{\alpha}}{D n} \Omega_{\alpha \gamma} n^{\gamma}\right) \mathbf{h}_{3}, \\
& \frac{d^{2} \phi}{d z^{2}}=\left\{\frac{D^{2} \phi^{\alpha}}{d z^{2}}-\phi^{\gamma} L_{\gamma}^{3} L^{3 \alpha}-U \phi^{\gamma} \Omega_{\gamma \delta} n^{\delta} L^{3 \alpha}-U \phi^{\gamma} \Omega_{\lambda}^{\alpha} n^{\lambda} L^{3}{ }_{\gamma}\right. \\
& -U^{2} \phi^{\gamma} \Omega_{\gamma \delta} n^{\delta} \Omega_{\lambda}^{\alpha} n^{\lambda}-2 \frac{d \phi^{3}}{d z} L^{3 \alpha}-2 U \frac{d \phi^{3}}{d z} \Omega_{\lambda}^{\alpha} n^{\lambda} \\
& \left.-\phi^{3}\left(\frac{D L^{3 \alpha}}{d z}+\frac{D U \Omega_{\lambda}^{\alpha}}{d z} n^{\lambda}-U\left(\frac{d U}{d m}+L_{n m}\right) \Omega_{\lambda}^{\alpha} m^{\lambda}\right)\right\} \mathbf{h}_{\alpha} \\
& +\left\{\frac{d^{2} \phi^{3}}{d z^{2}}-\phi^{3} L_{\gamma}^{3} L^{3 \gamma}-U \phi^{3} \Omega_{\gamma \delta} n^{\delta} L^{3 \gamma}-U \phi^{3} \Omega_{\lambda}^{\gamma} n^{\lambda} L_{\gamma}^{3}\right. \\
& -U^{2} \phi^{3} \Omega_{\gamma \delta} n^{\delta} \Omega_{\lambda}^{\gamma} n^{\lambda}+2 \frac{D \phi^{\alpha}}{d z} L_{\alpha}^{3}+2 U \frac{D \phi^{\alpha}}{d z} \Omega_{\alpha \beta} n^{\beta} \\
& \left.+\phi^{\alpha}\left(\frac{D L_{\alpha}^{3}}{d z}+\frac{D U \Omega_{\alpha \lambda}}{d z} n^{\lambda}-U\left(\frac{d U}{d m}+L_{n m}\right) \Omega_{\alpha \lambda} m^{\lambda}\right)\right\} \mathbf{h}_{3} \text {. }
\end{aligned}
$$

Using the general formula, from (2.13) and (2.14),

$$
\begin{aligned}
\frac{d\left(A^{\alpha} \mathbf{h}_{\alpha}+B \mathbf{h}_{3}\right)}{d z}= & \left\{\frac{D A^{\alpha}}{d z}-B\left(L^{3 \alpha}+U \Omega_{\beta}^{\alpha} n^{\beta}\right)\right\} \mathbf{h}_{\alpha} \\
& +\left\{A^{\alpha}\left(L^{3}{ }_{\alpha}+U \Omega_{\lambda \alpha} n^{\lambda}\right)+\frac{d B}{d z}\right\} \mathbf{h}_{3}
\end{aligned}
$$

further, partly temporal, directional derivatives can be calculated from (4.5) (because these expressions will not be differentiated again, terms whose jumps will vanish will be 
dropped):

$$
\frac{d^{3} \phi}{d z d m^{2}} \doteq 0 \doteq \frac{d^{3} \phi}{d z d m d n}
$$

and

$$
\begin{aligned}
\frac{d^{3} \phi}{d z d n^{2}} \doteq & \left(\frac{D^{3} \phi^{\alpha}}{d z D n^{2}}-\frac{d^{2} \phi^{3}}{d n^{2}}\left(L^{3 \alpha}+U \Omega_{\gamma}^{\alpha} n^{\gamma}\right)\right) h_{\alpha} \\
& +\left(\frac{d^{3} \phi^{3}}{d z d n^{2}}+\frac{D^{2} \phi^{\alpha}}{D n^{2}}\left(L_{\alpha}^{3}+U \Omega_{\alpha \gamma} n^{\gamma}\right)\right) h_{3} .
\end{aligned}
$$

From (4.22) differentiated again and with the usual simplification,

$$
\frac{d^{3} \phi}{d z^{2} d n} \doteq 0
$$

5. Partial derivatives. We take the second covariant derivative (3.1) of the vector field from [9],

$$
\begin{aligned}
\phi_{\mid \alpha \beta}= & \phi_{, \alpha \beta}-\phi_{. \delta}\left\{\begin{array}{c}
\delta \\
\alpha \beta
\end{array}\right\} \\
= & \left(\phi_{\mid \alpha \beta}^{\lambda}-\phi^{\sigma} \Omega_{\sigma \alpha} \Omega_{\beta}^{\lambda}-\phi_{, \alpha}^{3} \Omega_{\beta}^{\lambda}-\phi_{, \beta}^{3} \Omega_{\alpha}^{\lambda}-\phi^{3} \Omega_{\alpha \mid \beta}^{\lambda}\right) \mathbf{h}_{\lambda} \\
& +\left(\phi_{\mid \alpha}^{\lambda} \Omega_{\lambda \beta}+\left(\phi^{\lambda} \Omega_{\lambda \alpha}\right)_{\mid \beta}+\phi_{\mid \alpha \beta}^{3}-\phi^{3} \Omega_{\alpha}^{\lambda} \Omega_{\lambda \beta}\right) \mathbf{h}_{3} .
\end{aligned}
$$

From this we obtain directly, using (2.7) and (2.8) above,

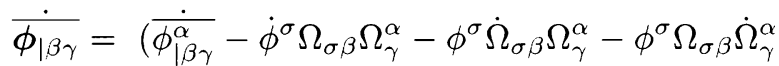

$$
\begin{aligned}
& -\phi_{\mid \beta}^{\delta} L^{3 \alpha} \Omega_{\delta \gamma}-\left(\phi^{\delta} \Omega_{\delta \beta}\right)_{\mid \gamma} L^{3 \alpha}+\phi_{\mid \beta \gamma}^{\delta} L_{\delta}^{\alpha}-\phi^{\sigma} L_{\delta}^{\alpha} \Omega_{\sigma \beta} \Omega_{\gamma}^{\delta} \\
& -\dot{\phi}_{, \beta}^{3} \Omega_{\gamma}^{\alpha}-\phi_{, \beta}^{3} \dot{\Omega}_{\gamma}^{\alpha}-\dot{\phi}_{, \gamma}^{3} \Omega_{\beta}^{\alpha}-\phi_{, \gamma}^{3} \dot{\Omega}_{\beta}^{\alpha}-\dot{\phi}^{3} \Omega_{\beta \mid \gamma}^{\alpha} \\
& \left.-\phi^{3} \overline{\Omega_{\beta \mid \gamma}^{\alpha}}-\phi_{\mid \beta \gamma}^{3} L^{3 \alpha}+\phi^{3} L^{3 \alpha} \Omega_{\beta}^{\delta} \Omega_{\delta \gamma}-\phi_{. \beta}^{3} L_{\delta}^{\alpha} \Omega_{\gamma}^{\delta}-\left(\phi^{3} \Omega_{\beta}^{\delta}\right)_{\mid \gamma} L_{\delta}^{\alpha}\right) \mathbf{h}_{\alpha} \\
& +\left(\phi_{\mid \beta \gamma}^{\delta} L_{\delta}^{3}-\phi^{\sigma} L_{\delta}^{3} \Omega_{\sigma \beta} \Omega_{\gamma}^{\delta}+\dot{\phi_{\mid \beta}^{\alpha}} \Omega_{\alpha \gamma}+\phi_{\mid \beta}^{\alpha} \dot{\Omega}_{\alpha \gamma}\right. \\
& +\dot{\phi_{\mid \gamma}^{\alpha}} \Omega_{\alpha \beta}+\phi_{\mid \gamma}^{\alpha} \dot{\Omega}_{\alpha \beta}+\dot{\phi}^{\alpha} \Omega_{\alpha \beta \mid \gamma}+\phi^{\alpha} \overline{\Omega_{\alpha \beta \mid \gamma}} \\
& \left.-\phi_{, \beta}^{3} L_{\delta}^{3} \Omega_{\gamma}^{\delta}-\left(\phi^{3} \Omega_{\beta}^{\delta}\right)_{\mid \gamma} L_{\delta}^{3}+\overline{\phi_{\mid \beta \gamma}^{3}}-\dot{\phi}^{3} \Omega_{\beta}^{\alpha} \Omega_{\alpha \gamma}-\phi^{3} \dot{\Omega}_{\beta}^{\alpha} \Omega_{\alpha \gamma}-\phi^{3} \Omega_{\beta}^{\alpha} \dot{\Omega}_{\alpha \gamma}\right) \mathbf{h}_{3} \text {. }
\end{aligned}
$$

In order to be able to compare this expression with one based on directional derivatives, three transformations need to be applied to it. All of the dotted $\phi$ terms except the two that formulas are sought for, $\frac{\dot{\phi_{\mid \beta \gamma}^{\alpha}}}{\text { and }} \overline{\phi_{\mid \beta \gamma}^{3}}$, must be replaced. This replacement is done for first derivatives by using the expressions (4.12) and, where an expression is a second derivative, using the expressions from which the second-order kinematic compatibility conditions come when jumps are taken, (4.11). The factor $\overline{\phi_{\mid \beta}^{\alpha}}$ can be dealt with exactly like $\dot{\phi}_{\mid \beta}^{\alpha}$ because the difference between the two expressions, according to Eq. (8.30) of [6], is $\phi^{\varepsilon}$ times a geometrical coefficient and so can be ignored here since its jump will vanish. The second transformation is to expand covariant derivatives in terms of directional derivatives using the formulas

$$
\phi_{\mid \beta}^{\alpha}=\frac{D \phi^{\alpha}}{D m} m_{\beta}+\frac{D \phi^{\alpha}}{D n} n_{\beta}, \quad \phi_{. \beta}^{3}=\frac{d \phi^{3}}{d m} m_{\beta}+\frac{d \phi^{3}}{d n} n_{\beta},
$$


and the same iterated and similar formulas applied to $\Omega_{\gamma}^{\alpha}, L^{3 \alpha}$, and $L^{\alpha}{ }_{\gamma}$. The third transformation is to insert components of the tangent and normal vectors $\mathbf{m}$ and $\mathbf{n}$ in each term except for $\frac{\dot{\phi}}{\phi_{\mid \beta \gamma}^{\alpha}}$ and $\overline{\phi_{\mid \beta \gamma}^{3}}$. This can be done by replacing each subscript $\beta$ (or $\gamma$ respectively) by a dummy suffix, say $\varepsilon$, and multiplying the term by $\delta_{\beta}^{\varepsilon}$ in the form $m^{\varepsilon} m_{\beta}+n^{\varepsilon} n_{\beta}$ (Eq. (6.25) of [6]). The result of these expansions is a substantial expression, which is not worth looking at and which was formed by the Mathematica package "Ricci" [7] running on a NeXT computer. (Without this combination of facilities, these calculations would not have been completed.)

We take the second partial derivative of $\phi$ with respect to time from [8],

$$
\begin{aligned}
\ddot{\phi}=\left(\ddot{\phi}^{\alpha}+2 \dot{\phi}^{\gamma} L_{\gamma}^{\alpha}+\phi^{\gamma} \dot{L}_{\gamma}^{\alpha}+\phi^{\lambda} L_{\lambda}^{\gamma} L_{\gamma}^{\alpha}-\phi^{\gamma} L_{\gamma}^{3} L^{3 \alpha}\right. \\
\left.-2 \dot{\phi}^{3} L^{3 \alpha}-\phi^{3} \dot{L}^{3 \alpha}-\phi^{3} L^{3 \gamma} L_{\gamma}^{\alpha}\right) \mathbf{h}_{\alpha} \\
+\left(\ddot{\phi}^{3}+2 \dot{\phi}^{\gamma} L_{\gamma}^{3}+\phi^{\gamma} \dot{L}_{\gamma}^{3}-\phi^{3} L^{3 \gamma} L_{\gamma}^{3}+\phi^{\lambda} L_{\lambda}^{\gamma} L_{\gamma}^{3}\right) \mathbf{h}_{3},
\end{aligned}
$$

and differentiate it partially with respect to $\theta^{\lambda}$ to obtain

$$
\begin{aligned}
\ddot{\phi}_{, \lambda}= & \left(\ddot{\phi}_{\mid \lambda}^{\alpha}+2 \dot{\phi}_{\mid \lambda}^{\gamma} L_{\gamma}^{\alpha}+2 \dot{\phi}^{\gamma} L_{\gamma \mid \lambda}^{\alpha}+\phi_{\mid \lambda}^{\gamma} \dot{L}_{\gamma}^{\alpha}+\phi^{\gamma} \dot{L}_{\gamma \mid \lambda}^{\alpha}+\phi_{\mid \lambda}^{\delta} L_{\delta}^{\gamma} L_{\gamma}^{\alpha}\right. \\
& +\phi^{\delta} L_{\delta \mid \lambda}^{\gamma} L_{\gamma}^{\alpha}+\phi^{\delta} L_{\delta}^{\gamma} L_{\gamma \mid \lambda}^{\alpha}-\phi_{\mid \lambda}^{\gamma} L_{\gamma}^{3} L^{3 \alpha}-\phi^{\gamma} L_{\gamma \mid \lambda}^{3} L^{3 \alpha} \\
& -\phi^{\gamma} L_{\gamma}^{3} L_{\mid \lambda}^{3 \alpha}-2 \dot{\phi}_{, \lambda}^{3} L^{3 \alpha}-2 \dot{\phi}^{3} L_{\mid \lambda}^{3 \alpha}-\phi_{, \lambda}^{3} \dot{L}^{3 \alpha} \\
& \left.-\phi^{3} \dot{L}_{\mid \lambda}^{3 \alpha}-\phi_{, \lambda}^{3} L^{3 \gamma} L_{\gamma}^{\alpha}-\phi^{3} L_{\mid \lambda}^{3 \gamma} L_{\gamma}^{\alpha}-\phi^{3} L^{3 \gamma} L_{\gamma \mid \lambda}^{\alpha}\right) \mathbf{h}_{\alpha} \\
& -\left(2 \dot{\phi}^{\gamma} L_{\gamma}^{3}+\phi^{\gamma} \dot{L}_{\gamma}^{3}+\phi^{\delta} L_{\delta}^{\gamma} L_{\gamma}^{3}+\ddot{\phi}^{3}-\phi^{3} L^{3 \gamma} L_{\gamma}^{3}\right) \Omega_{\lambda}^{\alpha} \mathbf{h}_{\alpha} \\
& +\left(\ddot{\phi}^{\alpha}+2 \dot{\phi}^{\gamma} L_{\gamma}^{\alpha}+\phi^{\gamma} \dot{L}_{\gamma}^{\alpha}+\phi^{\delta} L_{\delta}^{\gamma} L_{\gamma}^{\alpha}-\phi^{\gamma} L_{\gamma}^{3} L^{3 \alpha}-2 \dot{\phi}^{3} L^{3 \alpha}\right. \\
& \left.\quad-\phi^{3} \dot{L}^{3 \alpha}-\phi^{3} L^{3 \gamma} L_{\gamma}^{\alpha}\right) \Omega_{\alpha \lambda} \mathbf{h}_{3} \\
& +\left(2 \dot{\phi}_{\mid \lambda}^{\gamma} L_{\gamma}^{3}+2 \dot{\phi}^{\gamma} L_{\gamma \mid \lambda}^{3}+\phi_{\mid \lambda}^{\gamma} \dot{L}_{\gamma}^{3}+\phi^{\gamma} \dot{L}_{\gamma \mid \lambda}^{3}\right. \\
& +\phi_{\mid \lambda}^{\delta} L_{\delta}^{\gamma} L_{\gamma}^{3}+\phi^{\delta} L_{\delta \mid \lambda}^{\gamma} L_{\gamma}^{3}+\phi^{\delta} L_{\delta}^{\gamma} L_{\gamma \mid \lambda}^{3} \\
& \left.\quad+\ddot{\phi}_{, \lambda}^{3}-\phi_{, \lambda}^{3} L^{3 \gamma} L_{\gamma}^{3}-\phi^{3} L_{\mid \lambda}^{3 \gamma} L_{\gamma}^{3}-\phi^{3} L^{3 \gamma} L_{\gamma \mid \lambda}^{3}\right) \mathbf{h}_{3} .
\end{aligned}
$$

Since this expression will not again be differentiated, it is permissible to simplify it by the elimination of terms containing $\phi^{\alpha}$ and $\phi^{3}$, of which there are a goodly number. For comparison with the corresponding expression based on directional derivatives, as above the dotted $\phi$ terms except those sought, $\ddot{\phi}_{\mid \lambda}^{\alpha}$ and $\ddot{\phi}_{, \lambda}^{3}$ need to be replaced and the covariant derivatives expanded in directional derivatives. The result of this expansion is again a substantial expression, which exists only in the format of "Ricci". 
The third of the partial derivatives needed is obtained by differentiating the expression (5.4) with respect to time to obtain

$$
\begin{aligned}
& \dddot{\phi}=\left(\dddot{\phi}^{\alpha}+2 \ddot{\phi}^{\gamma} L_{\gamma}^{\alpha}+2 \dot{\phi}^{\gamma} \dot{L}_{\gamma}^{\alpha}+\dot{\phi}^{\gamma} \dot{L}_{\gamma}^{\alpha}+\phi^{\gamma} \ddot{L}_{\gamma}^{\alpha}+\dot{\phi}^{\lambda} L_{\lambda}^{\gamma} L_{\gamma}^{\alpha}\right. \\
& +\phi^{\lambda} \dot{L}_{\lambda}^{\gamma} L_{\gamma}^{\alpha}+\phi^{\lambda} L_{\lambda}^{\gamma} \dot{L}_{\gamma}^{\alpha}-\dot{\phi}^{\gamma} L^{3}{ }_{\gamma} L^{3 \alpha}-\phi^{\gamma} \dot{L}_{\gamma}^{3} L^{3 \alpha}-\phi^{\gamma} L^{3}{ }_{\gamma} \dot{L}^{3 \alpha}-2 \ddot{\phi}^{3} L^{3 \alpha} \\
& \left.-2 \dot{\phi}^{3} \dot{L}^{3 \alpha}-\dot{\phi}^{3} \dot{L}^{3 \alpha}-\phi^{3} \ddot{L}^{3 \alpha}-\dot{\phi}^{3} L^{3 \gamma} L^{\alpha}{ }_{\gamma}-\phi^{3} \dot{L}^{3 \gamma} L_{\gamma}^{\alpha}-\phi^{3} L^{3 \gamma} \dot{L}_{\gamma}^{\alpha}\right) \mathbf{h}_{\alpha} \\
& +\left(\ddot{\phi}^{3}+2 \dot{\phi}^{\gamma} L_{\gamma}^{3}+\phi^{\gamma} \dot{L}_{\gamma}^{3}-\phi^{3} L^{3 \gamma} L_{\gamma}^{3}+\phi^{\lambda} L_{\lambda}^{\gamma} L_{\gamma}^{3}\right)\left(-L^{3 \alpha} \mathbf{h}_{\alpha}\right) \\
& +\left(\ddot{\phi}^{\delta}+2 \dot{\phi}^{\gamma} L_{\gamma}^{\delta}+\phi^{\gamma} \dot{L}_{\gamma}^{\delta}+\phi^{\lambda} L_{\lambda}^{\gamma} L_{\gamma}^{\delta}-\phi^{\gamma} L^{3}{ }_{\gamma} L^{3 \delta}-2 \dot{\phi}^{3} L^{3 \delta}\right. \\
& \left.-\phi^{3} \dot{L}^{3 \delta}-\phi^{3} L^{3 \gamma} L_{\gamma}^{\delta}\right) L_{\delta}^{\alpha} \mathbf{h}_{\alpha} \\
& +\left(\ddot{\phi}^{\delta}+2 \dot{\phi}^{\gamma} L_{\gamma}^{\delta}+\phi^{\gamma} \dot{L}_{\gamma}^{\delta}+\phi^{\lambda} L_{\lambda}^{\gamma} L_{\gamma}^{\delta}-\phi^{\gamma} L_{\gamma}^{3} L^{3 \delta}-2 \dot{\phi}^{3} L^{3 \delta}\right. \\
& \left.-\phi^{3} \dot{L}^{3 \delta}-\phi^{3} L^{3 \gamma} L_{\gamma}^{\delta}\right) L^{3} \mathbf{h}_{3} \\
& +\left(\dddot{\phi}^{3}-\dot{\phi}^{3} L^{3 \gamma} L_{\gamma}^{3}-\phi^{3} \dot{L}^{3 \gamma} L_{\gamma}^{3}-\phi^{3} L^{3 \gamma} \dot{L}_{\gamma}^{3}+2 \ddot{\phi}^{\gamma} L^{3}{ }_{\gamma}+3 \dot{\phi}^{\gamma} \dot{L}^{3}{ }_{\gamma}\right. \\
& \left.+\phi^{\gamma} \ddot{L}_{\gamma}^{3}+\dot{\phi}^{\lambda} L_{\lambda}^{\gamma} L_{\gamma}^{3}+\phi^{\lambda} \dot{L}_{\lambda}^{\gamma} L_{\gamma}^{3}+\phi^{\lambda} L_{\lambda}^{\gamma} \dot{L}_{\gamma}^{3}\right) \mathbf{h}_{3} \text {. }
\end{aligned}
$$

Again, terms containing $\phi^{\alpha}$ and $\phi^{3}$ and (by (4.12)) $\dot{\phi}^{\alpha}$ and $\dot{\phi}^{3}$ can legitimately be removed to give the simplified form

$$
\dddot{\phi} \doteq\left(\dddot{\phi}^{\alpha}+3 \ddot{\phi}^{\gamma} L^{\alpha}{ }_{\gamma}-3 \ddot{\phi}^{3} L^{3 \alpha}\right) \mathbf{h}_{\alpha}+\left(\dddot{\phi}^{3}+3 \ddot{\phi}^{\gamma} L^{3}{ }_{\gamma}\right) \mathbf{h}_{3} .
$$

This expression then requires the same expansion as the previous partial derivative to produce an expression for comparison with the directional-derivative version of the same quantity. This has been done in "Ricci".

6. Compatibility conditions. The expression (3.19) for $\overline{\phi_{\mid \alpha \beta}}$ in terms of directional derivatives, with each vectorial expression expanded into coordinates by the formulas stated in Sec. two or derived in Sec. four and the now usual simplification, can be compared piece by piece with the same quantity computed in the fifth section to give expressions for $\frac{\dot{\phi}}{\phi_{\mid \beta \gamma}^{\alpha}}$ and $\frac{\dot{\phi}}{\phi_{\mid \beta \gamma}^{3}}$, which appear in that form in the section-five formulas. The resulting expressions, true only when jumps are taken, are:

$$
\begin{aligned}
\frac{\dot{\phi_{\mid \beta \gamma}^{\alpha}} \doteq \eta}{} & U \frac{D^{2} \phi^{\alpha}}{D n^{2}} m_{\beta} m_{\gamma} \\
& -\left(U \frac{D^{3} \phi^{\alpha}}{D m D n^{2}}+\frac{d U}{d m} \frac{D^{2} \phi^{\alpha}}{D n^{2}}\right)\left(m_{\beta} n_{\gamma}+n_{\beta} m_{\gamma}\right) \\
& +\left(\frac{D^{3} \phi^{\alpha}}{d z D n^{2}}-U \frac{D^{3} \phi^{\alpha}}{D n^{3}}+2 L_{n n} \frac{D^{2} \phi^{\alpha}}{D n^{2}}-L^{\alpha}{ }_{\delta} \frac{D^{2} \phi^{\delta}}{D n^{2}}\right) n_{\beta} n_{\gamma}
\end{aligned}
$$

and

$$
\begin{aligned}
& \overline{\phi_{\mid \beta \gamma}^{3}} \doteq \eta U \frac{d^{2} \phi^{3}}{d n^{2}} m_{\beta} m_{\gamma} \\
& -\left(U \frac{d^{3} \phi^{3}}{d m d n^{2}}+\frac{d U}{d m} \frac{d^{2} \phi^{3}}{d n^{2}}\right)\left(m_{\beta} n_{\gamma}+n_{\beta} m_{\gamma}\right) \\
& +\left(\frac{d^{3} \phi^{3}}{d z d n^{2}}-U \frac{d^{3} \phi^{3}}{d n^{3}}+2 L_{n n} \frac{d^{2} \phi^{3}}{d n^{2}}\right) n_{\beta} n_{\gamma} .
\end{aligned}
$$


When jumps are taken, a further simplification comes from using $\left(4.7_{2}\right)$ and (4.18):

$$
\begin{aligned}
{\left[\overline{\phi_{\mid \beta \gamma}^{3}}\right] \doteq } & \eta U \tilde{\tilde{\psi}}^{3} m_{\beta} m_{\gamma}-\frac{d U \tilde{\tilde{\psi}}^{3}}{d m}\left(m_{\beta} n_{\gamma}+n_{\beta} m_{\gamma}\right) \\
& +\left(\frac{d \tilde{\tilde{\psi}}^{3}}{d z}-U \tilde{\tilde{\psi}}^{3}+2\left[L_{n n} \frac{d^{2} \phi^{3}}{d n^{2}}\right]\right) n_{\beta} n_{\gamma}
\end{aligned}
$$

The tangential formula needs $\left(4.7_{1}\right)$ and (4.17). So

$$
\begin{aligned}
{\left[\frac{\dot{\phi}}{\mid \beta \gamma}\right] \doteq } & \eta U \tilde{\tilde{\psi}}^{\alpha} m_{\beta} m_{\gamma}-\frac{D U \tilde{\tilde{\psi}}^{\alpha}}{D m}\left(m_{\beta} n_{\gamma}+n_{\beta} m_{\gamma}\right) \\
& +\left(\frac{D \tilde{\tilde{\psi}}^{\alpha}}{d z}-U \tilde{\tilde{\tilde{\psi}}}+2\left[L_{n n} \frac{D^{2} \phi^{\alpha}}{D n^{2}}\right]-\left[L^{\alpha}{ }_{\delta} \frac{D^{2} \phi^{\delta}}{D n^{2}}\right]\right) n_{\beta} n_{\gamma}
\end{aligned}
$$

For the remaining formulas, it is necessary to have $\ddot{\phi}_{, \lambda}$ in terms of directional derivatives. This is obtained by partial differentiation with respect to $t$ of $\dot{\phi}_{, \lambda}$, which has already been used above. From (3.20), performing the partial differentiation using $d / d z-U d / d n$, the result is

$$
\begin{aligned}
& \ddot{\boldsymbol{\phi}}_{, \lambda}=\left\{\frac{d^{3} \boldsymbol{\phi}}{d z^{3} d m}-\frac{d U}{d z} \frac{d^{2} \boldsymbol{\phi}}{d m d n}-U \frac{d^{3} \boldsymbol{\phi}}{d z d m d n}+\frac{d}{d z}\left(L_{m m}-U \eta\right) \frac{d \boldsymbol{\phi}}{d m}\right. \\
& \left.+\left(L_{m m}-U \eta\right) \frac{d^{2} \phi}{d z d m}-\frac{d^{2} U}{d z d m} \frac{d \phi}{d n}-\frac{d U}{d m} \frac{d^{2} \phi}{d z d n}\right\} m_{\lambda} \\
& -U \frac{d}{d n}\left\{\frac{d^{2} \phi}{d z d m}-U \frac{d^{2} \phi}{d m d n}+\left(L_{m m}-U \eta\right) \frac{d \phi}{d m}-\frac{d U}{d m} \frac{d \phi}{d n}\right\} m_{\lambda} \\
& +\left\{\frac{d^{3} \phi}{d z^{2} d n}-\frac{d U}{d z} \frac{d^{2} \boldsymbol{\phi}}{d n^{2}}-U \frac{d^{3} \boldsymbol{\phi}}{d z d n^{2}}+\frac{d}{d z}\left(\frac{d U}{d m}+L_{n m}+L_{m n}+U \mu\right) \frac{d \phi}{d m}\right. \\
& \left.+\left(\frac{d U}{d m}+L_{n m}+L_{m n}+U \mu\right) \frac{d^{2} \phi}{d z d m}+\frac{d L_{n n}}{d z} \frac{d \phi}{d n}+L_{n n} \frac{d^{2} \phi}{d z d n}\right\} n_{\lambda} \\
& -U \frac{d}{d n}\left\{\frac{d^{2} \phi}{d z d n}-U \frac{d^{2} \phi}{d n^{2}}+\left(\frac{d U}{d m}+L_{n m}+L_{m n}+U \mu\right) \frac{d \phi}{d m}+L_{n n} \frac{d \phi}{d n}\right\} n_{\lambda} \\
& +\left\{\frac{d^{2} \phi}{d z d m}-U \frac{d^{2} \phi}{d m d n}+\left(L_{m m}-U \eta\right) \frac{d \phi}{d m}-\frac{d U}{d m} \frac{d \phi}{d n}\right\} \\
& \times\left\{L_{m m} m_{\lambda}+\left(L_{m n}+L_{n m}+\frac{d U}{d m}+U \mu\right) n_{\lambda}\right\} \\
& +\left\{\frac{d^{2} \phi}{d z d n}-U \frac{d^{2} \phi}{d n^{2}}+\left(\frac{d U}{d m}+L_{n m}+L_{m n}+U \mu\right) \frac{d \phi}{d m}+L_{n n} \frac{d \phi}{d n}\right\} \\
& \times\left\{-\left(U \mu+\frac{d U}{d m}\right) m_{\lambda}+L_{n n} n_{\lambda}\right\} .
\end{aligned}
$$

In the above expression, lines three and six can be replaced at a stroke using the pair of commutation relations (3.23). The expression can then be simplified by the removal of 
terms involving differentiation of $\phi$ only in the directions $\mathbf{m}, \mathbf{z}$, or a combination of them or of $d \phi / d n$ and its similar derivatives:

$$
\begin{aligned}
\ddot{\phi}_{. \lambda} \doteq & \left.-\frac{d U}{d z} \frac{d^{2} \phi}{d n^{2}}-U \frac{d^{3} \phi}{d z d n^{2}}\right) n_{\lambda} \\
& -U \frac{d^{2} \phi}{d n^{2}}\left\{-\left(U \mu+\frac{d U}{d m}\right) m_{\lambda}+L_{n n} n_{\lambda}\right\} \\
& -U\left\{\left(-U \frac{d^{3} \phi}{d m d n^{2}}-\frac{d U}{d m} \frac{d^{2} \phi}{d n^{2}}+\mu U \frac{d^{2} \phi}{d n^{2}}\right) m_{\lambda}\right. \\
& \left.+\left(\frac{d^{3} \phi}{d z d n^{2}}-U \frac{d^{3} \phi}{d n^{3}}+2 L_{n n} \frac{d^{2} \phi}{d n^{2}}\right) n_{\lambda}\right\}
\end{aligned}
$$

The two versions of $\ddot{\phi}_{, \lambda},(5.5)$ and (6.6), can now be compared component by component to produce the formulas for $\ddot{\phi}_{\mid \lambda}^{\alpha}$ and $\ddot{\phi}_{, \lambda}^{3}$. The only rule needed to produce compatibility between (5.5) and (6.6) is the expansion of the second fundamental form by (3.5) in (5.5). Again this comparison was done in "Ricci", the result in simplified form being

$$
\begin{aligned}
{\left[\ddot{\phi}_{\mid \lambda}^{\alpha}\right] \doteq } & {\left[2 U \frac{d U}{d m} \frac{D^{2} \phi^{\alpha}}{D n^{2}}+U^{2} \frac{D^{3} \phi^{\alpha}}{D m D n^{2}}\right] m_{\lambda} } \\
& +\left[U^{2} \frac{D^{3} \phi^{\alpha}}{D n^{3}}-2 U \frac{D^{3} \phi^{\alpha}}{d z D n^{2}}-\left(\frac{d U}{d z}+3 U L_{n n}\right) \frac{D^{2} \phi^{\alpha}}{D n^{2}}+2 U L^{\alpha}{ }_{\beta} \frac{D^{2} \phi^{\beta}}{D n^{2}}\right] n_{\lambda} .
\end{aligned}
$$

Using (4.71) and (4.17), the above formula simplifies to

$$
\begin{aligned}
{\left[\ddot{\phi}_{\mid \lambda}^{\alpha}\right] } & \doteq \frac{D U^{2} \tilde{\tilde{\psi}}^{\alpha}}{D m} m_{\lambda} \\
& +\left(U^{2} \tilde{\tilde{\psi}}^{\alpha}+\frac{D^{2} \tilde{\psi}^{\alpha}}{d z^{2}}-2 U \frac{D \tilde{\tilde{\psi}}^{\alpha}}{d z}-\left[\left(\frac{d U}{d z}+3 U L_{n n}\right) \frac{D^{2} \phi^{\alpha}}{D n^{2}}\right]+2 U\left[L^{\alpha}{ }_{\beta} \frac{D^{2} \phi^{\beta}}{D n^{2}}\right]\right) n_{\lambda}
\end{aligned}
$$

The other formula is

$$
\begin{aligned}
{\left[\ddot{\phi}_{, \lambda}^{3}\right] \doteq[2 U} & \left.\frac{d U}{d m} \frac{d^{2} \phi^{3}}{d n^{2}}+U^{2} \frac{d^{3} \phi^{3}}{d m d n^{2}}\right] m_{\lambda} \\
& +\left[U^{2} \frac{d^{3} \phi^{3}}{d n^{3}}-2 U \frac{d^{3} \phi^{3}}{d z d n^{2}}-\left(\frac{d U}{d z}+3 U L_{n n}\right) \frac{d^{2} \phi^{3}}{d n^{2}}\right] n_{\lambda}
\end{aligned}
$$

which simplifies, using $\left(4.7_{2}\right)$ and $(4.18)$, to

$$
\left[\ddot{\phi}_{, \lambda}^{3}\right] \doteq \frac{d U^{2} \tilde{\tilde{\psi}}^{3}}{d m} m_{\lambda}+\left(U^{2} \tilde{\tilde{\psi}}^{3}-2 U \frac{d \tilde{\tilde{\psi}}^{3}}{d z}-\left[\left(\frac{d U}{d z}+3 U L_{n n}\right) \frac{d^{2} \phi^{3}}{d n^{2}}\right]\right) n_{\lambda} .
$$

The third pair of formulas requires $\dddot{\phi}$ expanded in directional derivatives. This is easily obtained by differentiation of $\ddot{\phi}$ with respect to time using $d / d z$ and $d / d n$ :

$$
\dddot{\phi}=\frac{d \ddot{\phi}}{d z}-U \frac{d \ddot{\phi}}{d n}=\frac{d \ddot{\phi}}{d z}-U \ddot{\phi}_{, \lambda} n^{\lambda} \text {. }
$$


The second term is available as the coefficient of $n_{\lambda}$ in the expansion (6.6). The first term needs to be taken from Eq. (4.14) of [8],

$$
\begin{aligned}
\ddot{\phi}= & \frac{d^{2} \phi}{d z^{2}}-2 U \frac{d^{2} \phi}{d z d n}+U^{2} \frac{d^{2} \phi}{d n^{2}} \\
& -U\left(U \mu+\frac{d U}{d m}+L_{m n}+L_{n m}\right) \frac{d \phi}{d m}-\left(\frac{d U}{d z}+U L_{n n}\right) \frac{d \phi}{d n} .
\end{aligned}
$$

The expression wanted is

$$
\begin{aligned}
\frac{d \ddot{\boldsymbol{\phi}}}{d z}= & \frac{d^{3} \boldsymbol{\phi}}{d z^{3}}-2 \frac{d U}{d z} \frac{d^{2} \boldsymbol{\phi}}{d z d n}-2 U \frac{d^{3} \boldsymbol{\phi}}{d z^{2} d n}+2 U \frac{d U}{d z} \frac{d^{2} \boldsymbol{\phi}}{d n^{2}}+U^{2} \frac{d^{3} \boldsymbol{\phi}}{d z d n^{2}} \\
& -\frac{d}{d z}\left\{U\left(U \mu+\frac{d U}{d m}+L_{m n}+L_{n m}\right) \frac{d \phi}{d m}\right\} \\
& -\left(\frac{d^{2} U}{d z^{2}}+\frac{d U}{d z} L_{n n}+U \frac{d L_{n n}}{d z}\right) \frac{d \phi}{d n}-\left(\frac{d U}{d z}+U l_{n n}\right) \frac{d^{2} \boldsymbol{\phi}}{d z d n} \\
\doteq & 2 U \frac{d U}{d z} \frac{d^{2} \boldsymbol{\phi}}{d n^{2}}+U^{2} \frac{d^{3} \boldsymbol{\phi}}{d z d n^{2}} .
\end{aligned}
$$

The two versions (5.7) and (6.11) of $\dddot{\phi}$ are now comparable in "Ricci" with the derivatives of vectors all expanded in coordinates. The formulas produced are

$$
\left[\dddot{\phi}^{\alpha}\right] \doteq\left[-U^{3} \frac{D^{3} \phi^{\alpha}}{D n^{3}}+3 U^{2} \frac{D^{3} \phi^{\alpha}}{d z D n^{2}}+3 U\left(\frac{d U}{d z}+U L_{n n}\right) \frac{D^{2} \phi^{\alpha}}{D n^{2}}-3 U^{2} L^{\alpha}{ }_{\beta} \frac{D^{2} \phi^{\beta}}{D n^{2}}\right]
$$

and

$$
\left[\dddot{\phi}^{3}\right] \doteq\left[-U^{3} \frac{d^{3} \phi^{3}}{d n^{3}}+3 U^{2} \frac{d^{3} \phi^{3}}{d z d n^{2}}+3 U\left(\frac{d U}{d z}+U L_{n n}\right) \frac{d^{2} \phi^{3}}{d n^{2}}\right] .
$$

These simplify, using (4.7), (4.17), and (4.18), to

$$
\left[\dddot{\phi}^{\alpha}\right] \doteq-U^{3} \tilde{\tilde{\tilde{\psi}}}{ }^{\alpha}+3 U^{2} \frac{D \tilde{\tilde{\psi}}}{d z}+3 U\left[\left(\frac{d U}{d z}+U L_{n n}\right) \frac{D^{2} \phi^{\alpha}}{D n^{2}}\right]-3 U^{2}\left[L^{\alpha} \frac{D^{2} \phi^{\beta}}{D n^{2}}\right]
$$

and

$$
\left[\dddot{\phi}^{3}\right] \doteq-U^{3} \tilde{\tilde{\psi}}^{3}+3 U^{2} \frac{d \tilde{\tilde{\psi}}^{3}}{d z}+3 U\left[\left(\frac{d U}{d z}+U L_{n n}\right) \frac{d^{2} \phi^{3}}{d n^{2}}\right]
$$

7. Oblique trajectories. The usefulness of compatibility conditions with oblique trajectories, which can be chosen to be bicharacteristics of the partial differential equation being studied, has been well established. Nor is it difficult to find them. The operator $d / d \tau$ was defined in Sec. 2 in terms of $d / d z$ and $d / d m$. To produce compatibility conditions using $d / d \tau$ it is necessary only to replace $d / d z$ and its iterates in the results of the previous section by their equivalents in terms of $d / d \tau$ and $d / d m$,

$$
\frac{D \phi^{\alpha}}{d z}=\frac{D \phi^{\alpha}}{d \tau}-\gamma \frac{D \phi^{\alpha}}{D m}
$$


and correspondingly for $\phi^{3}$. When this formula is iterated, as it must be, commutation relations like those of Sec. 4 of [8] would be needed except that the terms in which they are needed are already assumed to vanish. Accordingly, the commutation relations (4.1) of [8] are not needed. When (7.1) is applied to expressions (6.1), (6.2), (6.7), (6.9), (6.14), and (6.15), they still simplify as they did in Sec. 6, and the results are the six kinematic compatibility conditions of third order for a surface vector field under the two simplifying assumptions set out in Sec. 1:

$$
\begin{aligned}
{\left[\overline{\phi_{\mid \beta \gamma}^{\alpha}}\right] \doteq \eta U \tilde{\tilde{\psi}}^{\alpha} m_{\beta} m_{\gamma}-\frac{D U \tilde{\tilde{\psi}}^{\alpha}}{D m}\left(m_{\beta} n_{\gamma}+n_{\beta} m_{\gamma}\right) } \\
\quad+\left(\frac{D \tilde{\tilde{\psi}}^{\alpha}}{d \tau}-\gamma \frac{D \tilde{\tilde{\psi}}^{\alpha}}{D m}-U \tilde{\tilde{\psi}}^{\alpha}+\left[2 L_{n n} \frac{D^{2} \phi^{\alpha}}{D n^{2}}-L^{\alpha}{ }_{\delta} \frac{D^{2} \phi^{\delta}}{D n^{2}}\right]\right) n_{\beta} n_{\gamma}
\end{aligned}
$$

and

$$
\begin{aligned}
{\left[\overline{\phi_{\mid \beta \gamma}^{3}}\right] \doteq \eta U } & \tilde{\tilde{\psi}^{3}} m_{\beta} m_{\gamma}-\frac{d U \tilde{\tilde{\psi}}^{3}}{d m}\left(m_{\beta} n_{\gamma}+n_{\beta} m_{\gamma}\right) \\
& +\left(\frac{d \tilde{\tilde{\psi}}^{3}}{d \tau}-\gamma \frac{d \tilde{\tilde{\psi}}^{3}}{d m}-U \tilde{\tilde{\tilde{\psi}}}^{3}+2\left[L_{n n} \frac{d^{2} \phi^{3}}{d n^{2}}\right]\right) n_{\beta} n_{\gamma}
\end{aligned}
$$

$$
\begin{aligned}
{\left[\ddot{\phi}_{\mid \lambda}^{\alpha}\right] } & \doteq \frac{D U^{2} \tilde{\tilde{\psi}}^{\alpha}}{D m} m_{\lambda} \\
& +\left\{U^{2} \tilde{\tilde{\tilde{\psi}}}{ }^{\alpha}-2 U \frac{D \tilde{\tilde{\psi}}^{\alpha}}{d \tau}+2 U \gamma \frac{D \tilde{\tilde{\psi}}^{\alpha}}{D m}+\left[2 U L^{\alpha}{ }_{\beta} \frac{D^{2} \phi^{\beta}}{D n^{2}}-\left(\frac{d U}{d z}+3 U L_{n n}\right) \frac{D^{2} \phi^{\alpha}}{D n^{2}}\right]\right\} n_{\lambda}
\end{aligned}
$$

and

$$
\left[\ddot{\phi}_{, \lambda}^{3}\right] \doteq \frac{d U^{2} \tilde{\tilde{\psi}}^{3}}{d m} m_{\lambda}+\left\{U^{2} \tilde{\tilde{\psi}}^{3}-2 U \frac{d \tilde{\tilde{\psi}}^{3}}{d \tau}+2 U \gamma \frac{d \tilde{\tilde{\psi}}^{3}}{d m}-\left[\left(\frac{d U}{d z}+3 U L_{n n}\right) \frac{d^{2} \phi^{3}}{d n^{2}}\right]\right\} n_{\lambda},
$$

and

$$
\begin{aligned}
{\left[\dddot{\phi}^{\alpha}\right] \doteq } & -U^{3} \tilde{\tilde{\tilde{\psi}}}{ }^{\alpha}+3 U^{2} \frac{D \tilde{\tilde{\psi}}^{\alpha}}{d \tau}-3 U^{2} \gamma \frac{D \tilde{\tilde{\psi}}^{\alpha}}{D m} \\
& +3 U\left[\left(\frac{d U}{d z}+U L_{n n}\right) \frac{D^{2} \phi^{\alpha}}{D n^{2}}-U L^{\alpha}{ }_{\beta} \frac{D^{2} \phi^{\beta}}{D n^{2}}\right]
\end{aligned}
$$

and

$$
\left[\dddot{\phi}^{3}\right] \doteq-U^{3} \tilde{\tilde{\psi}}^{3}+3 U^{2} \frac{d \tilde{\tilde{\psi}}^{3}}{d \tau}-3 U^{2} \gamma \frac{d \tilde{\tilde{\psi}}^{3}}{d m}+3 U\left[\left(\frac{d U}{d z}+U L_{n n}\right) \frac{d^{2} \phi^{3}}{d n^{2}}\right] .
$$

When $\mathbf{L}=0$, these six formulas reduce to those of [1].

Acknowledgments. I acknowledge support of this research from the University of Manitoba and the Natural Sciences and Engineering Research Council of Canada and help with various aspects of computing from Michael Doob, Jack Lee, David Mosscrop, Craig Platt, and Richard Tilley. 


\section{REFERENCES}

[1] H. Cohen and R. S. D. Thomas, Plane wave propagation and evolution for quasilinear hyperbolic systems, Util. Math. 24, 199-233 (1983)

[2] H. Cohen and R. S. D. Thomas, Transient waves in inhomogeneous isotropic elastic plates, Acta Mech. 53, 141-161 (1984)

[3] H. Cohen and R. S. D. Thomas, Transient waves in inhomogeneous anisotropic elastic plates, Acta Mech. 58, 41-57 (1986)

[4] H. Cohen and R. S. D. Thomas, Transient waves in inhomogeneous anisotropic elastic shells, Acta Mech. 77, 47-67 (1989)

[5] H. Cohen and R. S. D. Thomas, Transient waves in inhomogeneous elastic shells of variable thickness, Acta Mech. 83, 177-186 (1990)

[6] H. Cohen and C.-C. Wang, On compatibility conditions for singular surfaces, Arch. Rat. Mech. Anal. 80, 205-261 (1982)

[7] John M. Lee, Ricci: a Mathematica package for doing tensor calculations in differential geometry, 1992 (unpublished; available on WWW from http://www.wri.com/WWWocs/mathsource/ or by FTP from mathsource.wri.com)

[8] R. S. D. Thomas, Kinematic compatibility conditions for a three-dimensional vector field on a moving surface, Util. Math. 43, 7-32 (1993)

[9] R. S. D. Thomas, Geometric compatibility conditions of third order for a three-dimensional surface vector field, Algebras, Groups, and Geometries 10, 149-167 (1993)

[10] R. S. D. Thomas, Third-order kinematic compatibility conditions for a vector field on an embedded surface, Technical Report 1994-09-1, Department of Applied Mathematics, University of Manitoba, September, 1994

[11] T. Y. Thomas, Plastic flow and fracture in solids, Academic Press, New York, 1961

[12] C. Truesdell and R. A. Toupin, The classical field theories in Encyclopedia of Physics III/1 (S. Flügge, ed.), Springer, Berlin, 1960 